\title{
Uncertainty relations with the variance and the quantum Fisher information based on convex decompositions of density matrices
}

\author{
Géza Tóth ${ }^{1,2,3,4, *}$ and Florian Fröwis ${ }^{5}{ }^{5}$ \\ ${ }^{1}$ Department of Theoretical Physics, University of the Basque Country UPV/EHU, P.O. Box 644, E-48080 Bilbao, Spain \\ ${ }^{2}$ Donostia International Physics Center (DIPC), P.O. Box 1072, E-20080 San Sebastián, Spain \\ ${ }^{3}$ IKERBASQUE, Basque Foundation for Science, E-48013 Bilbao, Spain \\ ${ }^{4}$ Institute for Solid State Physics and Optics, Wigner Research Centre for Physics, P.O. Box 49, H-1525 Budapest, Hungary \\ ${ }^{5}$ Group of Applied Physics, University of Geneva, CH-1211 Geneva, Switzerland
}

(Received 13 October 2021; accepted 23 December 2021; published 31 January 2022;

corrected 30 September 2022 and 22 November 2022)

\begin{abstract}
We present several inequalities related to the Robertson-Schrödinger uncertainty relation. In all these inequalities, we consider a decomposition of the density matrix into a mixture of states, and use the fact that the Robertson-Schrödinger uncertainty relation is valid for all these components. By considering a convex roof of the bound, we obtain an alternative derivation of the relation in Fröwis et al. [Phys. Rev. A 92, 012102 (2015)], and we can also list a number of conditions that are needed to saturate the relation. We present a formulation of the Cramér-Rao bound involving the convex roof of the variance. By considering a concave roof of the bound in the Robertson-Schrödinger uncertainty relation over decompositions to mixed states, we obtain an improvement of the Robertson-Schrödinger uncertainty relation. We consider similar techniques for uncertainty relations with three variances. Finally, we present further uncertainty relations that provide lower bounds on the metrological usefulness of bipartite quantum states based on the variances of the canonical position and momentum operators for two-mode continuous variable systems. We show that the violation of well-known entanglement conditions in these systems discussed in Duan et al. [Phys. Rev. Lett. 84, 2722 (2000)] and Simon [Phys. Rev. Lett. 84, 2726 (2000)] implies that the state is more useful metrologically than certain relevant subsets of separable states. We present similar results concerning entanglement conditions with angular momentum operators for spin systems.
\end{abstract}

DOI: 10.1103/PhysRevResearch.4.013075

\section{INTRODUCTION}

Quantum Fisher information (QFI) is a central quantity of quantum metrology, a field that is concerned with metrological tasks in which the quantumness of the system plays an essential role [1-4]. One of the most fundamental scenarios in quantum metrology is estimating the small parameter $\theta$ in the unitary dynamics

$$
\varrho_{\theta}=e^{-i B \theta} \varrho e^{i B \theta},
$$

where $B$ is the Hamiltonian of the dynamics, $\varrho$ is the initial state, $\varrho_{\theta}$ is the final state of the evolution, and we set $\hbar=1$ for simplicity. By carrying out measurements on $\varrho_{\theta}$, we aim to estimate $\theta$ from the distribution of the outcomes of the measurement. The quantum Cramér-Rao inequality gives a lower bound on the precision of the estimation for any measurement

$$
(\Delta \theta)^{2} \geqslant \frac{1}{m F_{Q}[\varrho, B]},
$$

where $F_{Q}[\varrho, B]$ is the QFI and $m$ is the number of independent repetitions [5-8].

\footnotetext{
*toth@alumni.nd.edu; http://www.gtoth.eu
}

Published by the American Physical Society under the terms of the Creative Commons Attribution 4.0 International license. Further distribution of this work must maintain attribution to the author(s) and the published article's title, journal citation, and DOI.
At the center of attention lies the question how noise can affect the precision of the estimation [9] and what the ultimate limit of the precision is in realistic scenarios [10,11]. We add that a driving force behind the development in quantum metrology are recent experiments in quantum optical systems, such as cold gases and cold trapped ions, which are possible due to the rapid technological advancement in the field [12-15]. The experiments with the squeezed-light-enhanced gravitational wave detector GEO 600 [16-18] are highlights in the applications of quantum-enhanced sensitivity.

Recently, the QFI was discovered to play an important role in quantum information theory, in particular, in the theory of quantum entanglement [19]. It turns out that, in linear interferometers, entanglement is needed to surpass the shot-noise limit in precision corresponding to product states $[1-4,20]$. It has been shown that the larger the QFI, the larger the depth of entanglement the state must posses [21,22]. Beside the entanglement depth, there are further quantities that can give a more detailed information about the structure of the multipartite entanglement $[23,24]$, which turn out to be strongly connected to the QFI [25]. In general, the QFI can be used to detect multipartite entanglement, which has been done in several experiments [26-28]. Apart from entanglement theory, the QFI has also been used to define what it means that a superposition is macroscopically quantum [29,30] and to bound the speed of a quantum evolution [31,32], and it plays a role even in the quantum Zeno effect [33,34]. Finally, the QFI offers a 
powerful characterization of the prepared quantum state, for which it is calculated even from tomographic data [35]. It has been shown that this type of characterization is superior to computing the fidelity with respect to the ideal state for usual state reconstruction schemes [36].

Recent findings show that the QFI is the convex roof of the variance, apart from a constant factor [37,38]. This again connects quantum metrology to quantum information science where convex roofs often appear in the theory of entanglement measures $[39,40]$. Density matrices have an infinite number of convex decompositions. This is a feature of quantum mechanics not present in classical physics. So far, this fact is appreciated mostly in quantum information science; however, it can also be used as a powerful tool in other areas of quantum physics.

Finally, the QFI appears in various quantum uncertainty relations. In these relations, the error propagation formula defined as

$$
(\Delta \theta)_{A}^{2}=\frac{(\Delta A)^{2}}{\left|\partial_{\theta}\langle A\rangle\right|^{2}}
$$

plays a central role [41]. The uncertainty of the estimate is given by Eq. (3) divided by $m$, the number of independent repetitions, if the distribution of the measurement results fulfills certain reasonable requirements and $m$ is sufficiently large $[7,42,43]$. Then, from the Cramér-Rao bound (2), one can derive [41], for example, the Heisenberg-Robertson uncertainty relation [44,45], time-energy uncertainty relations [46-49], and squeezing inequalities [20,50]. The optimization of Eq. (3) over a given set of operators has been considered [51].

In this paper we use the knowledge that the QFI is, apart from a constant factor, the convex roof of the variance to obtain inequalities valid for all quantum states and to obtain entanglement criteria. First, we give a simple proof of a tighter version of the Heisenberg-Robertson uncertainty relation $[41,52]$, also giving conditions for saturation. We show ways to strengthen the Heisenberg-Robertson uncertainty relation. We derive the Cramér-Rao bound such that the bound is given by a convex roof. We derive a relation with two variances and a QFI. We also present entanglement conditions with the QFI.

Our paper is organized as follows. In Sec. II we summarize important properties of the QFI and the variance. In Sec. III we discuss recent finding connecting the QFI to convex roofs. In Sec. IV we present inequalities derived from the RobertsonSchrödinger uncertainty relation based on convex roofs. In Sec. $\mathrm{V}$ we present an improvement on the same inequality based on concave roofs. In Sec. VI we present uncertainty relations with variances and the QFI. In Sec. VII we present a simple relation and use it to rederive some of our results. We also derive further inequalities with the variance and the QFI. In Sec. VIII we show how to relate the violation of some entanglement conditions to the metrological usefulness of the quantum state.

\section{IMPORTANT PROPERTIES OF THE QFI}

In this section we briefly summarize the basic literature about the QFI. The properties we list will be used later in our calculations.
Most importantly, the QFI is convex, i.e.,

$$
F_{Q}\left[\varrho_{\mathrm{m}}, B\right] \leqslant p F_{Q}\left[\varrho_{1}, B\right]+(1-p) F_{Q}\left[\varrho_{2}, B\right],
$$

where the mixture is defined as

$$
\varrho_{\mathrm{m}}=p \varrho_{1}+(1-p) \varrho_{2} .
$$

Here lies an important similarity between the QFI and entanglement measures: neither of the two can increase under mixing.

The QFI appearing in the Cramér-Rao bound Eq. (2) is defined as [5-8,53]

$$
F_{Q}[\varrho, A]=2 \sum_{k, l} \frac{\left(\lambda_{k}-\lambda_{l}\right)^{2}}{\lambda_{k}+\lambda_{l}}|\langle k|A| l\rangle|^{2},
$$

where the density matrix has the eigendecomposition

$$
\varrho=\sum_{k} \lambda_{k}|k\rangle\langle k| \text {. }
$$

From Eq. (6), it follows that the QFI can be bounded from above by the variance

$$
F_{Q}[\varrho, B] \leqslant 4(\Delta B)_{\varrho}^{2},
$$

where equality holds if $\varrho$ is pure [7].

The Cramér-Rao bound (2) defines the achievable largest precision of parameter estimation; however, it is not clear what has to be measured to reach this precision bound. An optimal measurement can be carried out if we measure in the eigenbasis of the symmetric logarithmic derivative $\mathcal{L}[7,8]$. This operator is defined such that it can be used to describe the quantum dynamics of the system with the equation

$$
\frac{d \varrho_{\theta}}{d \theta}=\frac{1}{2}\left(\mathcal{L} \varrho_{\theta}+\varrho_{\theta} \mathcal{L}\right) .
$$

Unitary dynamics are generally given by the von Neumann equation with the Hamiltonian $B$

$$
\frac{d \varrho_{\theta}}{d \theta}=i\left(\varrho_{\theta} B-B \varrho_{\theta}\right) .
$$

The operator $\mathcal{L}$ can be found based on knowing that the righthand side of Eq. (9) must be equal to the right-hand side of Eq. (10):

$$
i[\varrho, B]=\frac{1}{2}\{\varrho, \mathcal{L}\} .
$$

Hence, the symmetric logarithmic derivative can be expressed with a simple formula as

$$
\mathcal{L}=2 i \sum_{k, l} \frac{\lambda_{k}-\lambda_{l}}{\lambda_{k}+\lambda_{l}}|k\rangle\langle l|\langle k|B| l\rangle,
$$

where $\lambda_{k}$ and $|k\rangle$ are the eigenvalues and eigenvectors, respectively, of the density matrix $\varrho$. Based on Eqs. (6) and (12), the symmetric logarithmic derivative can be used to obtain the QFI as

$$
F_{Q}[\varrho, B]=\operatorname{Tr}\left(\varrho \mathcal{L}^{2}\right)=(\Delta \mathcal{L})^{2} .
$$

In the second equality in Eq. (13), we used that

$$
\langle\mathcal{L}\rangle_{\varrho}=0,
$$

which can be seen based on Eqs. (7) and (12). 


\section{DEFINING THE QUANTUM FISHER INFORMATION WITH CONVEX ROOFS}

The quantum Fisher information has been connected to convex roofs that are based on an optimization over convex decompositions of the density matrix [37,38]. Let us consider a density matrix of the form

$$
\varrho=\sum_{k} p_{k}\left|\psi_{k}\right\rangle\left\langle\psi_{k}\right|,
$$

where $p_{k}>0$ and $\sum_{k} p_{k}=1$. Note that the pure states $\left|\psi_{k}\right\rangle$ are not required to be pairwise orthogonal, and Eq. (15) is not an eigendecomposition of the density matrix. Then it can be shown that the QFI is the convex roof of the variance times four $[37,38]$,

$$
F_{Q}[\varrho, B]=4 \min _{\left\{p_{k},\left|\psi_{k}\right\rangle\right\}} \sum_{k} p_{k}(\Delta B)_{\psi_{k}}^{2},
$$

where $\left\{p_{k},\left|\psi_{k}\right\rangle\right\}$ refers to a decomposition of $\varrho$ of the type Eq. (15). In other words, we already knew that the QFI is convex, but Eq. (16) implies that it is the smallest convex function that equals four times the variance for pure states. For further analysis on the convexity of the QFI, see Ref. [54].

Equation (16) has also been used in derivations concerning the continuity of the QFI [55], or finding efficient ways to bound it from below based on few measurements [56]. It has been used in constructing entanglement conditions in Ref. [57]. Finally, it has also been used in finding a bound on

$$
V(\varrho, A)=(\Delta A)^{2}-F_{Q}[\varrho, A] / 4
$$

based on the purity of $\varrho$ [58].

A related result is that the variance is the concave roof of itself,

$$
(\Delta A)_{\varrho}^{2}=\max _{\left\{p_{k},\left|\psi_{k}\right\rangle\right\}} \sum_{k} p_{k}(\Delta A)_{\psi_{k}}^{2} .
$$

This property of the variance is relatively easy to show $[37,38]$. For the proof, one has to demonstrate that there is always a decomposition of the type Eq. (15) such that

$$
\langle A\rangle_{\psi_{k}}=\langle A\rangle_{\varrho}
$$

for all $k$. Similar decompositions for correlation matrices have been considered in Refs. [59,60].

The statements of Eqs. (16) and (18) can be concisely reformulated as follows. For any decomposition $\left\{p_{k},\left|\psi_{k}\right\rangle\right\}$ of the density matrix $\varrho$ we have

$$
\frac{1}{4} F_{Q}[\varrho, A] \leqslant \sum_{k} p_{k}(\Delta A)_{\psi_{k}}^{2} \leqslant(\Delta A)_{\varrho}^{2},
$$

where the upper and the lower bounds are both tight.

Note that the QFI has been connected to convex roofs in another context, via purifications $[10,11,61,62]$. The basic idea is that the QFI can easily be computed for pure states and a unitary dynamics. For the more general case of mixed states and noisy dynamics we can still deal with pure states if we add an ancillary system and consider the purification of the noisy dynamics.

Finally, let us discuss that relations given in Eqs. (16) and (18) remain the same if we optimize over decompositions to a mixture of density matrices instead of decompositions to a mixture of pure states. Let us consider a decomposition of $\varrho$ to a mixture of density matrices [63]

$$
\varrho=\sum_{k} p_{k} \varrho_{k}
$$

Due to the fact that the QFI and the variance are convex and concave, respectively, in density matrices, the inequalities

$$
\frac{1}{4} F_{Q}[\varrho, A] \leqslant \sum_{k} p_{k}(\Delta A)_{\varrho_{k}}^{2} \leqslant(\Delta A)_{\varrho}^{2}
$$

hold. However, we already know that decompositions to a mixture of pure states can saturate both inequalities in Eq. (20). Thus, obtaining the convex and concave roofs over decompositions to mixed states will lead to same values that we obtain in Eqs. (16) and (18).

Concerning the relation for the QFI given in Eq. (16), we can add the following. If we calculate the convex roof of a quantity that is concave in density matrices, then the result of a minimization over all pure-state decompositions will coincide with the result of a minimization over all mixed-state decompositions. The reason is that if a concave function is minimized over a convex set, then it takes its minima on the extreme points of the set. Similarly, if we calculate the concave roof of a quantity that is convex in density matrices, then the result of a maximization over all pure-state decompositions will coincide with the result of a maximization over all mixed-state decompositions.

\section{UNCERTAINTY RELATIONS BASED ON A CONVEX ROOF OVER DECOMPOSITIONS IN THE ROBERTSON-SCHRÖDINGER INEQUALITY}

The Robertson-Schrödinger uncertainty is a fundamentally important uncertainty relation in quantum physics [45]. Hence, there is a strong interest in deriving further relations from it and in looking for possible improvements [64-67]. In this section we present a simple method to obtain further uncertainty relations based on the optimization over the decompositions of density matrices. We rederive the improved Heisenberg-Robertson inequality presented in Ref. [41]. We discuss some implications of the Cramér-Rao bound and determine which states saturate the inequality.

\section{A. Simple proof for the improved Heisenberg-Robertson inequality presented in Ref. [41]}

The Robertson-Schrödinger inequality is defined as

$$
(\Delta A)_{\varrho}^{2}(\Delta B)_{\varrho}^{2} \geqslant \frac{1}{4}\left|L_{\varrho}\right|^{2},
$$

where the lower bound is given by

$$
L_{\varrho}=\sqrt{\left|\langle\{A, B\}\rangle_{\varrho}-2\langle A\rangle_{\varrho}\langle B\rangle_{\varrho}\right|^{2}+\left|\langle C\rangle_{\varrho}\right|^{2}},
$$

$\{A, B\}=A B+B A$ is the anticommutator, and we use the definition

$$
C=i[A, B] .
$$

First, let us examine the convexity properties of the bound on the right-hand side of Eq. (23), which we need later. One can show that $L_{\varrho}$ is neither convex nor concave in $\varrho$. Let us 
consider a concrete example, the mixed two-qubit state with a decomposition

$$
\begin{array}{ll}
p_{1}=1 / 2, & \left|\psi_{1}\right\rangle=|00\rangle, \\
p_{2}=1 / 2, & \left|\psi_{2}\right\rangle=|11\rangle,
\end{array}
$$

and the operators

$$
\begin{aligned}
& A=\sigma_{z} \otimes \mathbb{1}, \\
& B=\mathbb{1} \otimes \sigma_{z} .
\end{aligned}
$$

For these, we have $C=0, L_{\psi_{1}}=L_{\psi_{2}}=0$, while $L_{\varrho}=1$. Simple algebra shows that $L_{\varrho}>p_{1} L_{\psi_{1}}+p_{2} L_{\psi_{2}}$ holds. Let us consider another concrete example, the mixed state with a decomposition

$$
\begin{array}{ll}
p_{1}=1 / 2, & \left|\psi_{1}\right\rangle=(|00\rangle+|11\rangle) / \sqrt{2}, \\
p_{2}=1 / 2, & \left|\psi_{2}\right\rangle=(|01\rangle+|10\rangle) / \sqrt{2},
\end{array}
$$

and the same operators given in Eq. (27). For these, $C=0$, $L_{\psi_{1}}=L_{\psi_{2}}=1$, while $L_{\varrho}=0$. Hence, $L_{\varrho}<p_{1} L_{\psi_{1}}+p_{2} L_{\psi_{2}}$ holds.

Since $L_{\varrho}$ is neither convex nor concave in $\varrho$, we will now consider a decomposition of the density matrix to mixed states $\varrho_{k}$ as given in Eq. (21) instead of a decomposition to pure states. For such a decomposition, for all $\varrho_{k}$ the RobertsonSchrödinger inequality given in Eq. (23) holds. From this fact and with the simple inequality presented in Appendix A, we arrive at

$$
\left[\sum_{k} p_{k}(\Delta A)_{\varrho_{k}}^{2}\right]\left[\sum_{k} p_{k}(\Delta B)_{\varrho_{k}}^{2}\right] \geqslant \frac{1}{4}\left[\sum_{k} p_{k} L_{\varrho_{k}}\right]^{2} .
$$

At this point it is important to know that the inequality in Eq. (29) is valid for any decomposition of the density matrix of the type given in Eq. (21). Moreover, we should remember that the three sums are over the same decomposition of the density matrix.

Let us try to obtain inequalities with the variance and the QFI. For that, we can choose the decomposition such that

$$
\sum_{k} p_{k}(\Delta B)_{\varrho_{k}}^{2}
$$

is minimal and equals $F_{Q}[\varrho, B] / 4$ given in Eq. (16). Due to the concavity of the variance we also know that

$$
\sum_{k} p_{k}(\Delta A)_{\varrho_{k}}^{2} \leqslant(\Delta A)^{2}
$$

Hence, it follows for the product of the variance of $A$ and the QFI $F_{Q}[\varrho, B]$ that

$$
(\Delta A)_{\varrho}^{2} F_{Q}[\varrho, B] \geqslant\left(\sum_{k} p_{k} L_{\varrho_{k}}\right)^{2} .
$$

In order to use Eq. (32), we need to know the decomposition that minimizes Eq. (30). We can have a inequality where we do not need to know that decomposition:

$$
(\Delta A)_{\varrho}^{2} F_{Q}[\varrho, B] \geqslant\left(\min _{\left\{p_{k}, \varrho_{k}\right\}} \sum_{k} p_{k} L_{\varrho_{k}}\right)^{2} .
$$

On the right-hand side of Eq. (33), the bound is defined based on a convex roof. The right-hand side of Eq. (33) is not larger than the right-hand side of Eq. (32). We can also see that on the right-hand side of Eq. (33) there is a minimization over mixed-state decompositions. Based on Sec. III, there is always an optimal pure-state decomposition such that Eq. (30) is minimal and equals $F_{Q}[\varrho, B] / 4$. Thus, we can also have a valid inequality with an optimization over pure-state decompositions of the type given in Eq. (15):

$$
(\Delta A)_{\varrho}^{2} F_{Q}[\varrho, B] \geqslant\left(\min _{\left\{p_{k},\left|\psi_{k}\right\rangle\right\}} \sum_{k} p_{k} L_{\psi_{k}}\right)^{2} .
$$

The right-hand side of Eq. (34) is not smaller than the righthand side of Eq. (33). Hence, in the remaining part of the section we will work with pure-state decompositions rather than mixed-state decompositions.

Let us now try to find a lower bound for the inequality that is easier to compute, while possibly being smaller than the bound in Eq. (34). One could first think of using $\left|L_{\varrho}\right|^{2}$ as a lower bound; however, it is not convex in $\varrho$, as we have discussed. Based on Eq. (24), the relation

$$
L_{\psi_{k}} \geqslant\left|\langle C\rangle_{\psi_{k}}\right|
$$

holds. Based on Eq. (34) and Eq. (35), we can obtain the inequality

$$
(\Delta A)_{\varrho}^{2} F_{Q}[\varrho, B] \geqslant\left(\min _{\left\{p_{k},\left|\psi_{k}\right\rangle\right\}} \sum_{k} p_{k}\left|\langle C\rangle_{\psi_{k}}\right|\right)^{2},
$$

Using well-known properties of the absolute value we get

$$
\sum_{k} p_{k}\left|\langle C\rangle_{\psi_{k}}\right| \geqslant\left|\sum_{k} p_{k}\langle C\rangle_{\psi_{k}}\right| \equiv\left|\langle C\rangle_{\varrho}\right|,
$$

and with that we arrive at the improved Heisenberg-Robertson uncertainty proved by Fröwis et al. [41]:

$$
(\Delta A)_{\varrho}^{2} F_{Q}[\varrho, B] \geqslant\left|\langle C\rangle_{\varrho}\right|^{2} .
$$

Due to the relation between the variance and the QFI given in Eq. (8), the left-hand side of Eq. (38) is never larger than the left-hand side of the Heisenberg-Robertson uncertainty.

Based on these, we find the following.

Observation 1. The improved Heisenberg-Robertson inequality (38) can be saturated only if all of the following conditions are fulfilled.

(i) There is a decomposition $\left\{p_{k},\left|\psi_{k}\right\rangle\right\}$ that minimizes the weighted sum of the subensemble variances for the operator $B$, hence

$$
\frac{1}{4} F_{Q}[\varrho, B]=\sum_{k} p_{k}(\Delta B)_{\psi_{k}}^{2} .
$$

We also need that it maximizes the weighted sum of the subensemble variances for the operator $A$, and hence

$$
(\Delta A)_{\varrho}^{2}=\sum_{k} p_{k}(\Delta A)_{\psi_{k}}^{2}
$$

(ii) If the decomposition maximizes the weighted sum of the subensemble variances for the operator $A$, then Eq. (19) holds. [See explanation after Eq. (18).] 
(iii) Moreover, Eq. (35) must be saturated for every $k$. Hence, the equality

$$
\frac{1}{2}\langle\{A, B\}\rangle_{\psi_{k}}-\langle A\rangle_{\psi_{k}}\langle B\rangle_{\psi_{k}}=0
$$

must hold. In this case, we also have

$$
(\Delta[A+B])_{\psi_{k}}^{2}=(\Delta A)_{\psi_{k}}^{2}+(\Delta B)_{\psi_{k}}^{2} .
$$

(iv) Equation (29) is saturated for pure-state decompositions only if for the subensemble variances the equations

$$
\begin{aligned}
& (\Delta A)_{\psi_{k}}^{2}=(\Delta A)_{\psi_{l}}^{2}, \\
& (\Delta B)_{\psi_{k}}^{2}=(\Delta B)_{\psi_{l}}^{2}
\end{aligned}
$$

hold for all $k, l$. (See Appendix A.)

(v) For such an optimal decomposition, for every $k$,

$$
\left|\langle C\rangle_{\psi_{k}}\right|=\left|\langle C\rangle_{\varrho}\right|
$$

which is trivially fulfilled if $C$ is a constant. (See Appendix A.) This is the case, for example, if $A$ and $B$ are the position and momentum operators, $x$ and $p$, of a bosonic mode.

\section{B. Implications for the Cramér-Rao bound}

In this section we will show that the precision of parameter estimation is bounded from below by an expression with the convex roof of the variance.

Let us first define a relevant notion. The error propagation formula is given in Eq. (3). Using the fact that the dynamics is unitary, we have

$$
\left|\partial_{\theta}\langle A\rangle\right|=|\langle C\rangle|,
$$

where $C$ is defined in Eq. (25) (see, e.g., Ref. [41]). Hence

$$
(\Delta \theta)_{A}^{2}=\frac{(\Delta A)^{2}}{|\langle C\rangle|^{2}} .
$$

Then, the precision of the estimation is bounded as

$$
(\Delta \theta)^{2} \geqslant \frac{1}{m} \min _{A}(\Delta \theta)_{A}^{2},
$$

where $m$ is the number of independent repetitions. In the large $m$ limit, if certain further conditions are fulfilled, Eq. (47) can be saturated [68].

Based on these and on Sec. IV A, we arrive at

$$
(\Delta \theta)_{A}^{2} \geqslant \frac{1}{4 \min _{\left\{p_{k},\left|\psi_{k}\right\rangle\right\}}\left[\sum_{k} p_{k}(\Delta B)_{\psi_{k}}^{2}\right]} .
$$

Using Eqs. (46) and (47), we get a lower bound on the precision of parameter estimation

$$
(\Delta \theta)^{2} \geqslant \frac{1}{m} \times \frac{1}{4 \min _{\left\{p_{k},\left|\psi_{k}\right\rangle\right\}}\left[\sum_{k} p_{k}(\Delta B)_{\psi_{k}}^{2}\right]} .
$$

We have just derived a form of the Cramér-Rao bound that contains the convex roof of the variance. On the right-hand side of Eq. (49) we write intentionally the expression with the convex roof, rather than the QFI, to stress that our derivation did not use the formula given in Eq. (6) for the QFI. The Cramér-Rao bound in Eq. (49) can be saturated only if the conditions of Observation 1 are fulfilled for some $A$.
Note that we did not prove that there is an $A$ for every $B$ and $\varrho$ such that the bound in Eq. (49) can be saturated, which would be necessary to prove that the Cramér-Rao bound can be reached.

Note also that we did not consider POVM measurements, which would be the more general case [7]. However, it is known that it is always possible to saturate the Cramér-Rao bound by von Neumann measurements [2].

\section{Sufficient condition for saturating the bound}

In this section, for completeness, we present a concise sufficient condition that Eq. (38) is saturated. Similar statements have been discussed in Refs. [41,69-71]. This is relevant for us, since it is connected to the conditions for saturation given in Observation 1. We use the theory of the symmetric logarithmic derivative described in Sec. II.

Observation 2 . If the equality

$$
i[\varrho, B]=\frac{1}{2}\{\varrho, c A\}
$$

holds, then Eq. (38) is saturated. Here $c \neq 0$ is a real constant.

Proof. Equation (50) implies that $c A$ equals the symmetric logarithmic derivative $\mathcal{L}$; see Eq. (11). Let us then substitute $c A$ by $\mathcal{L}$ in Eq. (38). Then it follows that $[7,8]$

$$
F_{Q}[\varrho, B]=\left\langle\mathcal{L}^{2}\right\rangle_{\varrho}=c^{2}(\Delta A)_{\varrho}^{2},
$$

and moreover simple algebra yields

$$
\langle i[A, B]\rangle_{\varrho}=\frac{1}{c} \operatorname{Tr}(i[\mathcal{L}, B] \varrho)=\frac{1}{c}\left\langle\mathcal{L}^{2}\right\rangle_{\varrho}=c(\Delta A)_{\varrho}^{2} .
$$

In the last equality in Eq. (52) we used Eq. (14). Consequently, the left-hand side and the right-hand side of Eq. (38) are equal, and the state saturates the inequality. Moreover, the two terms of the product on the left-hand side of Eq. (38) are equal to each other, that is, $(\Delta A)^{2}=F_{Q}[\varrho, B]$.

Observation 2 is related to the known relation

$$
(\Delta \mathcal{L})^{2} F_{Q}[\varrho, B]=\left|\langle i[\mathcal{L}, B]\rangle_{\varrho}\right|^{2},
$$

where $\mathcal{L}$ is defined in Eq. (12), and on the left-hand side of Eq. (53), the two terms in the product are equal to each other.

Note that as a consequence, the equality in Eq. (50) implies that conditions in Observation 1 are fulfilled. Moreover, based on Observation 2, we can find additional constraints on the subsensemble variances given in Observation 1. If we compute the trace of both sides of Eq. (50) we arrive at

$$
\langle A\rangle_{\varrho}=0 .
$$

From Observation 1 (ii) follows that there is a similar statement for all subensembles

$$
\langle A\rangle_{\psi_{k}}=0 .
$$

Moreover, based on Observation 1 (iii), we obtain a relation about the commutator of $A$ and $B$ as

$$
\langle\{A, B\}\rangle_{\psi_{k}}=0
$$

which is again valid for all subensembles. 


\section{IMPROVEMENT ON THE ROBERTSON- SCHRÖDINGER INEQUALITY BASED ON A CONCAVE ROOF OVER DECOMPOSITIONS}

In this section we show an improvement of the RobertsonSchrödinger inequality. We start from the fact that Eq. (29) is valid for any decomposition of the density matrix to mixed components $\varrho_{k}$. Hence, due to the concavity of the variance follows that

$$
(\Delta A)^{2}(\Delta B)^{2} \geqslant \frac{1}{4}\left(\sum_{k} p_{k} L_{\varrho_{k}}\right)^{2} .
$$

Based on these, we can find the following.

Observation 3. For quantum states, the following inequality holds:

$$
(\Delta A)_{\varrho}^{2}(\Delta B)_{\varrho}^{2} \geqslant \frac{1}{4}\left(\max _{\left\{p_{k}, \varrho_{k}\right\}} \sum_{k} p_{k} L_{\varrho_{k}}\right)^{2},
$$

where $L_{\varrho}$ is defined in Eq. (24). On the right-hand side of Eq. (58), we have a concave roof. The relation in Eq. (58) is saturated by all single-qubit mixed states, and it is stronger than the Robertson-Schrödinger inequality given in Eq. (23).

Proof. By taking the maximum of the bound on the righthand side of Eq. (57) over mixed-state decompositions, we arrive at the inequality Eq. (58).

Let us examine the single-qubit case in detail. Let us take the operators

$$
A=\sigma_{x}, \quad B=\cos \alpha \sigma_{x}+\sin \alpha \sigma_{y},
$$

which is the most general case, apart for trivial rotations of the coordinate system. We characterize the state by the Bloch vector elements $\left\langle\sigma_{l}\right\rangle$ for $l=x, y, z$. Substituting these in the bound in the Robertson-Schrödinger inequality given in Eq. (23) we obtain for pure states

$$
\begin{aligned}
\frac{1}{4}\left|L_{\psi}\right|^{2}= & {\left[\cos \alpha\left(\Delta \sigma_{x}\right)_{\psi}^{2}-\sin \alpha\left\langle\sigma_{x}\right\rangle_{\psi}\left\langle\sigma_{y}\right\rangle_{\psi}\right]^{2} } \\
& +\sin ^{2} \alpha\left\langle\sigma_{z}\right\rangle_{\psi}^{2} .
\end{aligned}
$$

Substituting $\left\langle\sigma_{z}\right\rangle_{\psi}^{2}=1-\left\langle\sigma_{x}\right\rangle_{\psi}^{2}-\left\langle\sigma_{y}\right\rangle_{\psi}^{2}$ into Eq. (60) we arrive at

$$
\begin{aligned}
\frac{1}{4}\left|L_{\psi}\right|^{2}= & \left(\Delta \sigma_{x}\right)_{\psi}^{2}\left[\cos ^{2} \alpha\left(\Delta \sigma_{x}\right)^{2}\right. \\
& \left.-\sin 2 \alpha\left\langle\sigma_{x}\right\rangle_{\psi}\left\langle\sigma_{y}\right\rangle_{\psi}+\sin ^{2} \alpha\left(\Delta \sigma_{y}\right)^{2}\right] .
\end{aligned}
$$

Simple algebra leads to

$$
\frac{1}{4}\left|L_{\psi}\right|^{2}=(\Delta A)_{\psi}^{2}(\Delta B)_{\psi}^{2} .
$$

Hence, all pure states saturate Eq. (58).

We will now show that for every single-qubit mixed state and every single-qubit operator the inequality in Eq. (58) is saturated. If we can find a decomposition $\left\{p_{k}, \varrho_{k}\right\}$, such that

$$
(\Delta X)_{\varrho}^{2}=(\Delta X)_{\varrho_{k}}^{2}
$$

for $X=A, B$ and all $k$, then this is sufficient to have equality in Eq. (58). The following decomposition has this property. We imagine the Bloch sphere with a vector representing an arbitrary $\varrho$ and a straight line that goes through $\varrho$ and that is parallel to the $z$ axis. All states along this line within the Bloch sphere have the same expectation values for $\sigma_{x}$ and $\sigma_{y}$ as $\varrho$, hence also the same variances. Therefore, we choose a decomposition of $\varrho$ with the pure states at the points where the line intersects with the Bloch sphere. With this, we have equality in Eq. (58).

Let us now prove that the Robertson-Schrödinger inequality in Eq. (58) is stronger than the Robertson-Schrödinger inequality given in Eq. (23). First, the right-hand side of Eq. (58) is never smaller than the right-hand side of the Robertson-Schrödinger inequality given in Eq. (23). This is evident since one of the possible decompositions is

$$
p_{1}=1, \quad \varrho_{1}=\varrho .
$$

Second, let us now consider a concrete example when the bound in Eq. (58) is higher than the bound in the RobertsonSchrödinger inequality given in Eq. (23). For instance, let us consider the completely mixed state

$$
\varrho=\mathbb{1} / 2
$$

and the operators given in Eq. (59). Since we found that the inequality in Eq. (58) is saturated by all single-qubit mixed states, it is also saturated for the state given in Eq. (65) and the right-hand side of Eq. (58) equals 1 . The right-hand side of the Robertson-Schrödinger inequality given in Eq. (23) is zero.

Let us consider now higher dimensional systems. Here not all pure states saturate the inequality given in Eq. (23). As an example, let us consider qutrit states, and the operators

$$
A=J_{x}, \quad B=J_{y} .
$$

First, we show a simple method that never gives a bound lower than the bound in Eq. (23), and often it gives a higher bound. Let us consider the bound based on Eq. (57) using the eigendecomposition of $\varrho$ given in Eq. (7):

$$
(\Delta A)_{\varrho}^{2}(\Delta B)_{\varrho}^{2} \geqslant \frac{1}{4}\left(\sum_{k} \lambda_{k} L_{|k\rangle}\right)^{2} .
$$

Since $L_{\varrho}$ is not convex in $\varrho$, the bound in the inequality given in Eq. (67) might be smaller than the bound in the RobertsonSchrödinger relation given in Eq. (23).

We will now present a relation for which the bound is never smaller than in Eq. (23). Let us consider the unnormalized states

$$
\sigma_{k}=\varrho-\lambda_{k}|k\rangle\langle k|
$$

for $k=1,2,3$. Here $\sigma_{k}$ is a mixture of the two basis vectors orthogonal to $|k\rangle$. Then we define the probabilities and normalized states

$$
p_{k}=\operatorname{Tr}\left(\sigma_{k}\right), \quad \varrho_{k}=\sigma_{k} / p_{k} .
$$

Using $\varrho_{k}$, we decompose the density matrix as

$$
\varrho=\lambda_{k}|k\rangle\langle k|+p_{k} \varrho_{k},
$$

where $k \in\{1,2,3\}$. With these we define the quantity

$$
\tilde{L}_{k}=\lambda_{k} L_{|k\rangle}+p_{k} L_{\varrho_{k}} .
$$

Then we obtain an inequality

$$
(\Delta A)_{\varrho}^{2}(\Delta B)_{\varrho}^{2} \geqslant \frac{1}{4} K^{2} .
$$



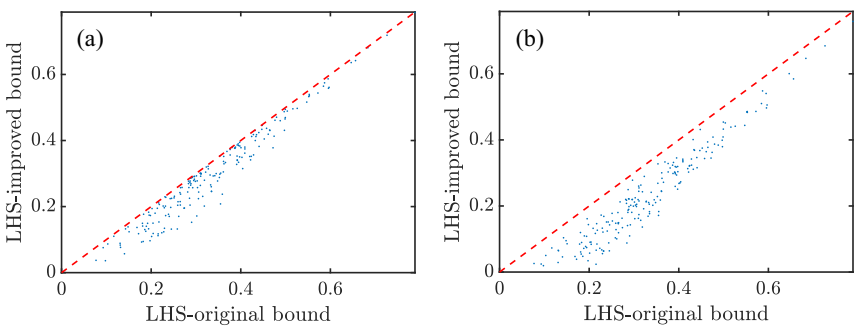

FIG. 1. (a) The left-hand side (LHS) minus the right-hand side (RHS) for the Robertson-Schrödinger inequality (23) vs LHS-RHS for Eq. (58), taking the eigendecomposition of the density matrix and using the inequality given in Eq. (72). Points that are below the dashed line correspond to quantum states for which the bound improved. Even if our method is simple, the improvement is significant. We generated 200 random states. (b) The same for the concave roof obtained numerically.

where the variable in the bound is defined as

$$
K=\max \left(\sum_{k} \lambda_{k} L_{|k\rangle}, \tilde{L}_{1}, \tilde{L}_{2}, \tilde{L}_{3}, L_{\varrho}\right),
$$

and $\max \left(a_{1}, a_{2}, a_{3}, \ldots\right)$ denotes the maximum of $a_{k}$. Since $K \geqslant L_{\varrho}$, the bound in Eq. (72) is never smaller than the bound in the Robertson-Schrödinger inequality given in (23). We will see that it is often larger. We considered all the possible ways to group the eigenvectors into groups and form mixed states from them. Such ideas can straightforwardly be generalized to larger dimensions, where we need to consider more partitions of the eigenvectors.

Next, we will test the uncertainty relation given in Eq. (58) numerically. We generate random single-qutrit states [72]. We calculate the usual bound in the Robertson-Schrödinger inequality given in Eq. (23). We test the simple method given in Eq. (72) that give improved bounds. The results can be seen in Fig. 1(a). Note that typically we do not find the best possible bound, but still this simple technique often leads to an improvement and the new bound is significantly larger than the old one. There are also numerical methods to compute the concave roof in Eq. (58), described in Appendix B. Based on that we carry out numerical optimization over mixed-state decompositions. The results can be seen in Fig. 1(b). Even if the numerical search might not find the global maximum, but something smaller, we found a valid lower bound on the left-hand side of Eq. (58).

\section{UNCERTAINTY RELATIONS WITH SEVERAL VARIANCES AND THE QFI}

In this section we derive uncertainty relations with the QFI, and one or more variances. This provides a lower bound on the QFI based on variances of angular momentum operators.

\section{A. Sum of two variances}

Ideas similar to the ones in Sec. IV work even if we have uncertainty relations that are the sum of two variances. For example, for a continuous variable system

$$
(\Delta x)^{2}+(\Delta p)^{2} \geqslant 1
$$

holds, where $x$ and $p$ are the position and momentum operators. This must be valid for any state, including pure states. Hence, for any decompositions of the density matrix it follows that

$$
\sum_{k} p_{k}(\Delta x)_{\psi_{k}}^{2}+\sum_{k} p_{k}(\Delta p)_{\psi_{k}}^{2} \geqslant 1
$$

For one of the two operators, say, for $p$, we can choose the decomposition that leads to the minimal value for the average variance, i.e., the QFI over four. Then, since $\sum_{k} p_{k}(\Delta x)_{\psi_{k}}^{2} \leqslant$ $(\Delta x)^{2}$ holds, it follows that

$$
(\Delta x)^{2}+\frac{1}{4} F_{Q}[\varrho, p] \geqslant 1 .
$$

Note that this could be obtained more directly from the uncertainty relation in Eq. (38) using

$$
X+Y \geqslant 2 \sqrt{X Y},
$$

for $X, Y \geqslant 0$, but we intended to demonstrate the key idea of the next sections.

\section{B. Lower bound on the QFI}

Similar reasoning works for the uncertainty relations for the sum of three variances. Let us start from the relation for pure states

$$
\left(\Delta J_{x}\right)^{2}+\left(\Delta J_{y}\right)^{2}+\left(\Delta J_{z}\right)^{2} \geqslant j,
$$

where $J_{l}$ are the spin components fulfilling

$$
J_{x}^{2}+J_{y}^{2}+J_{z}^{2}=j(j+1) \mathbb{1} .
$$

Due to the concavity of the variance, the inequality in Eq. (78) holds also for mixed states. We can improve this relation. From the inequality given in Eq. (78), following the ideas of Sec. VI A, we arrive at

$$
\left(\Delta J_{x}\right)^{2}+\left(\Delta J_{y}\right)^{2}+\frac{1}{4} F_{Q}\left[\varrho, J_{z}\right] \geqslant j .
$$

Equation (80) is a stronger relation than Eq. (78). The lefthand side of Eq. (80) is never smaller than left-hand side of Eq. (78). The difference between the two is given in Eq. (17). This quantity has been studied in Ref. [58]. It is zero for pure states and largest for the state

$$
\frac{1}{2}\left(|-j\rangle\left\langle-\left.j\right|_{z}+\mid+j\right\rangle\left\langle+\left.j\right|_{z}\right) .\right.
$$

For this case, for the $z$-component of the spin we have $\left(\Delta J_{z}\right)^{2}=j^{2}$ and $F_{Q}\left[\varrho, J_{z}\right]=0$, while for the variance of the $x$-component we have $\left(\Delta J_{x}\right)^{2}=j / 2$. Thus, the state given in Eq. (81) saturates the inequality in Eq. (80).

Based on these, we arrive at the following observation.

Observation 4. For a spin- $j$ particle, the following inequality bounds from below the metrological usefulness of the state

$$
F_{Q}\left[\varrho, J_{z}\right] \geqslant 4 j-4\left(\Delta J_{x}\right)^{2}-4\left(\Delta J_{y}\right)^{2}=: B_{F Q} .
$$

Let us now examine whether the bound in Eq. (82) can be improved. It is known that Eq. (78) is saturated by all pure $\mathrm{SU}(2)$ coherent states or spin-coherent states, which are defined as

$$
|s\rangle=U|+j\rangle_{z}
$$




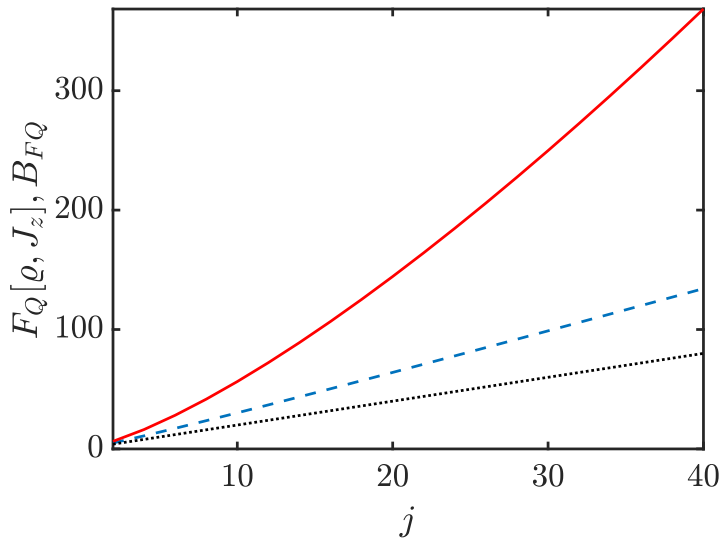

FIG. 2. Planar squeezed states. (solid) The QFI and (dashed) our lower bound $B_{F Q}$ given in Eq. (82) for planar squeezed states for a range of $j$. (dotted) As a reference, we plot the QFI corresponding to the state fully polarized in the $x$-direction.

where the unitary is given as

$$
U=e^{-i \vec{c} \vec{J}},
$$

where $\vec{c}$ is a three-vector of numbers and $\vec{J}=\left(J_{x}, J_{y}, J_{z}\right)$. Hence, the inequality given in Eq. (82) is also saturated by all such states, and the bound is optimal.

The inequality in Eq. (82) bounds the QFI from below based on variances. Such a bound can be very useful in some situations, since we do not need to carry out a metrological task to get information about $F_{Q}\left[\varrho, J_{z}\right]$. Let us now consider some quantum states, and compare the bound given by Eq. (82) to the QFI of those states. Our first example will be planar squeezed states [73]. Such states saturate the uncertainty relation

$$
\left(\Delta J_{x}\right)^{2}+\left(\Delta J_{y}\right)^{2} \geqslant C_{j},
$$

where for the bound

$$
C_{\frac{1}{2}}=\frac{1}{4}, \quad C_{1}=\frac{7}{16}
$$

holds, while for higher $j$ 's the bound is obtained numerically in Ref. [73]. Note that planar squeezed states minimize the left-hand side of Eq. (85) such that their mean spin is not zero. Thus, they are different from the states that minimize $\left\langle J_{x}^{2}\right\rangle+\left\langle J_{y}^{2}\right\rangle$.

Based on the inequalities given in Eqs. (82) and (85), for planar squeezed states we have

$$
F_{Q}\left[\varrho, J_{z}\right] \geqslant B_{F Q}=4\left(j-C_{j}\right) .
$$

In Eq. (87) the value of $B_{F Q}$ approaches $4 j$ since for large $j$ we have [73]

$$
C_{j} \ll j .
$$

In Fig. 2 we plotted the QFI vs our lower bound for planar squeezed states for various $j$ 's.

We will present another class of states for which our bound on the QFI can be useful. We will consider the state $|j\rangle_{x}$ squeezed in the $y$-direction. Spin-squeezed states can be obtained as the ground states of the Hamiltonian [74]

$$
H_{\mathrm{sq}}(\lambda)=J_{y}^{2}-\lambda J_{x} \text {. }
$$

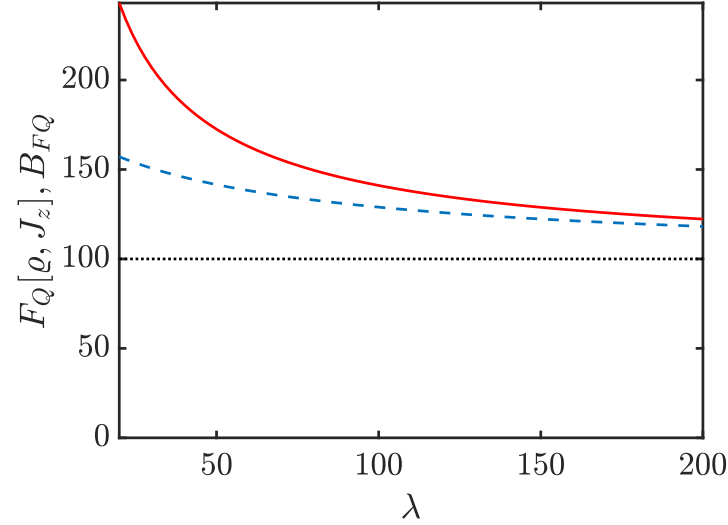

FIG. 3. Spin-squeezed states. (solid) The QFI and (dashed) our lower bound $B_{F Q}$ defined in Eq. (82) for spin-squeezed states with $j=50$. The spin-squeezed states are obtained as the ground states of Eq. (89) for a range of $\lambda$. (dotted) As a reference, we plot the QFI corresponding to the state fully polarized in the $x$-direction.

For $\lambda=\infty$, the ground state is $|j\rangle_{x}$, the state fully polarized in the $x$-direction. For $0<\lambda<\infty$, it is a state spin squeezed along the $y$-direction. When the state becomes squeezed along the $y$-direction, the sum of the two variances in Eq. (82) starts to decrease. Then, due to Eq. (82), the QFI has to increase and the state becomes more useful for metrology. In Fig. 3 we plotted the right-hand side and the left-hand side of Eq. (82) for a range of $\lambda$ for $j=50$. Our lower bound is quite close to the QFI for states with an almost maximal spin.

Next, we will determine what the largest precision is for $\mathrm{SU}(2)$ coherent states or spin-coherent states defined in Eq. (83). It is easy to show that $F_{Q}\left[\varrho, J_{z}\right]$ is maximal for $|j\rangle_{x}$, the $\mathrm{SU}(2)$ coherent state pointing into in the $x$-direction. For that state, we have

$$
F_{Q}\left[|j\rangle_{x}, J_{z}\right]=2 j, \quad\left(\Delta J_{x}\right)^{2}=0, \quad\left(\Delta J_{y}\right)^{2}=j / 2 .
$$

Due to the convexity of the QFI, for the mixtures of SU(2) coherent states

$$
F_{Q}\left[\varrho_{\mathrm{SU}(2)-\text { mixture }}, J_{z}\right] \leqslant 2 j
$$

holds. Any state that violates Eq. (91) is more useful metrologically than a mixture of $\mathrm{SU}(2)$ coherent states. Both in Fig. 2 and in Fig. 3, we plot a line corresponding to the bound in the inequality given in Eq. (91).

Finally, let us generalize these ideas to more than three operators. Let us consider the following relation for pure states:

$$
\sum_{n=1}^{d^{2}-1}\left(\Delta G_{n}\right)^{2}=4 j,
$$

where $G_{n}$ are the $\mathrm{SU}(d)$ generators fulfilling

$$
\operatorname{Tr}\left(G_{k} G_{l}\right)=2 \delta_{k l},
$$

and $d=2 j+1$ is the dimension of the qudit (see e.g., Ref. [75]). Due to the concavity of the variance it follows that for mixed states [75]

$$
\sum_{n=1}^{d^{2}-1}\left(\Delta G_{n}\right)^{2} \geqslant 4 j .
$$


We can even have a better relation based on the discussion before.

Observation 5. For a spin- $j$ particle, the following inequality bounds from above the metrological usefulness of the state:

$$
\frac{1}{4} F_{Q}\left[\varrho, G_{1}\right]+\sum_{n=2}^{d^{2}-1}\left(\Delta G_{n}\right)^{2} \geqslant 4 j
$$

\section{ALTERNATIVE DERIVATION BASED ON CONVEXITY ARGUMENTS}

In this section we present a simple idea that can be used to rederive some of the previous results. The derivation becomes much shorter, while the conditions for saturating the inequalities are not so easy to obtain. We also derive further inequalities with the variance and the QFI. Part of the section is a summary of already existing results, which we are connecting to the methods of the paper.

Observation 6. Let us consider a relation

$$
(\Delta A)_{\varrho}^{2} \geqslant g(\varrho),
$$

which is true for pure states. If $g(\varrho)$ is convex in density matrices, then

$$
\frac{1}{4} F_{Q}[\varrho, A] \geqslant g(\varrho)
$$

holds for mixed states. If $g(\varrho)$ is not convex in $\varrho$, the inequality

$$
\frac{1}{4} F_{Q}[\varrho, A] \geqslant \min _{\left\{p_{k},\left|\psi_{k}\right\rangle\right\}} \sum_{k} p_{k} g\left(\left|\psi_{k}\right\rangle\right)
$$

still holds.

Proof. On the left-hand side of the inequality in Eq. (97) there is the QFI of $\varrho$ over four. Based on Eq. (16), we know that it is a convex roof, that is, the largest convex function that equals $(\Delta A)_{\varrho}^{2}$ for all pure states. If $g(\varrho)$ is convex in $\varrho$, then, on the right-hand side of Eq. (97), there is an expression that is never larger than the left-hand side for pure states. Even if $g(\varrho)$ is not convex in $\varrho$, then the right-hand side of Eq. (98) is still convex in $\varrho$.

Using Observation 6, the inequality with the product of the variance and the QFI given in Eq. (34) can be proved as follows. We will use the ideas of Ref. [76] to convert relations with the product of uncertainties to relations with the sum of uncertainties. Based on Eq. (77), we know that

$$
\alpha(\Delta A)^{2}+\beta(\Delta B)^{2} \geqslant 2 \sqrt{\alpha \beta} \sqrt{(\Delta A)^{2}(\Delta B)^{2}}
$$

holds for all $\alpha, \beta \geqslant 0$. From the product uncertainty relation given in Eq. (23) and from Eq. (99) it follows that for the weighted sum of the variances

$$
\alpha(\Delta A)^{2}+\beta(\Delta B)^{2} \geqslant \sqrt{\alpha \beta} L_{\varrho}
$$

holds for all $\alpha, \beta \geqslant 0$. From Eq. (100), we can get a relation with a quantity on the right-hand side that is convex in the density matrix:

$$
\alpha(\Delta A)^{2}+\beta(\Delta B)^{2} \geqslant \sqrt{\alpha \beta} \min _{\left\{p_{k},\left|\psi_{k}\right\rangle\right\}} \sum_{k} p_{k} L_{\psi_{k}} .
$$

Let us rewrite Eq. (101) as

$$
\beta(\Delta B)^{2} \geqslant \sqrt{\alpha \beta} \min _{\left\{p_{k},\left|\psi_{k}\right\rangle\right\}}\left(\sum_{k} p_{k} L_{\psi_{k}}\right)-\alpha(\Delta A)^{2} .
$$

Now on the left-hand side we have a variance, while the right-hand side is convex in the state. Using Observation 5, we arrive at

$$
\beta \frac{1}{4} F_{Q}[\varrho, B] \geqslant \sqrt{\alpha \beta} \min _{\left\{p_{k},\left|\psi_{k}\right\rangle\right\}}\left(\sum_{k} p_{k} L_{\psi_{k}}\right)-\alpha(\Delta A)^{2} .
$$

Hence, we arrive at a relation with the weighted sum of the variance and the QFI:

$$
\alpha(\Delta A)^{2}+\beta \frac{1}{4} F_{Q}[\varrho, B] \geqslant \sqrt{\alpha \beta} \min _{\left\{p_{k},\left|\psi_{k}\right\rangle\right\}}\left(\sum_{k} p_{k} L_{\psi_{k}}\right) .
$$

From the fact that the inequality in Eq. (103) holds for all $\alpha, \beta \geqslant 0$ follows the inequality with the product of the variance and the QFI given in Eq. (34).

Using Observation 6, the uncertainty relation with two variances and the QFI given in Eq. (82) can be proved as follows. The uncertainty relation with three variances in Eq. (78) can be rewritten as

$$
\left(\Delta J_{x}\right)^{2} \geqslant j-\left(\Delta J_{y}\right)^{2}-\left(\Delta J_{z}\right)^{2},
$$

which is of the form given in Eq. (96), since its right-hand side is convex in $\varrho$ and the left-hand side is a variance. Hence, the inequality in Eq. (82) can be rederived.

Next, we prove a general bound on the metrological usefulness of a quantum state based on its spin length using Observation 6.

Observation 7. The metrological usefulness of a state is bounded with the spin length as

$$
F_{Q}\left[\varrho, J_{x}\right] \geqslant 4 j F_{j}\left(\left\langle J_{z}\right\rangle / j\right),
$$

where $F_{j}(X)$ is a convex function defined as

$$
F_{j}(X)=\min _{\varrho:\left\langle J_{z}\right\rangle=X j} \frac{\left(\Delta J_{x}\right)^{2}}{j} .
$$

In particular, if $\left\langle J_{z}\right\rangle \neq 0$, then $F_{Q}\left[\varrho, J_{x}\right]>0$.

Proof. For the components of the angular momentum for a particle with spin- $j[74]$

$$
\left(\Delta J_{x}\right)^{2} \geqslant j F_{j}\left(\left\langle J_{z}\right\rangle / j\right)
$$

holds. Using Observation 6, we can obtain an inequality for the QFI

$$
\frac{1}{4} F_{Q}\left[\varrho, J_{x}\right] \geqslant j F_{j}\left(\left\langle J_{z}\right\rangle / j\right) .
$$

Based on the definition in Eq. (107), it is clear that if $\left\langle J_{z}\right\rangle>0$, then $F_{j}\left(\left\langle J_{z}\right\rangle / j\right)>0$. Hence, based on the relation in Eq. (106) follows that $F_{Q}\left[\varrho, J_{x}\right]>0$. Thus, if the $z$-component of the angular momentum has a nonzero expectation value, then the state can be used for metrology with the Hamiltonian $J_{x}$.

Usually, the function $F_{j}(X)$ is computed by looking for the ground state $\left|\Psi_{\lambda, \lambda_{2}}\right\rangle$ of the Hamiltonian [74]

$$
H_{\lambda, \lambda_{2}}=J_{x}^{2}-\lambda J_{z}-\lambda_{2} J_{x},
$$

where $\lambda$ and $\lambda_{2}$ play the role of Lagrange multipliers. In particular, we need the ground state of the Hamiltonian given 
in Eq. (110) for which $\left\langle J_{z}\right\rangle$ equals a given value and $\left(\Delta J_{x}\right)^{2}$ is minimal, that is,

$$
F_{j}(X)=\min _{\lambda, \lambda_{2}:\left\langle J_{z}\right\rangle_{\left|\Psi_{\lambda, \lambda_{2}}\right\rangle}=X j}\left(\Delta J_{x}\right)_{\left|\Psi_{\lambda, \lambda_{2}}\right\rangle}^{2} / j .
$$

In this way the minimization is over two real parameters, rather than over a quantum state. Such a calculation has been used to obtain a lower bound on the variance $\left(\Delta J_{x}\right)^{2}$, if the expectation value $\left\langle J_{z}\right\rangle$ is constrained to be a given constant [74]. For an integer $j$, the state minimizing $\left(\Delta J_{x}\right)^{2}$ has $\left\langle J_{x}\right\rangle=0$, thus $\lambda_{2}$ can be omitted from the Hamiltonian in Eq. (110). Later it has also been shown that with such a procedure we get a lower bound on $\frac{1}{4} F_{Q}\left[\varrho, J_{x}\right][56]$.

Observation 8. Let us consider a relation

$$
\sum_{n=1}^{N_{A}}\left(\Delta A_{n}\right)_{\varrho}^{2} \geqslant g(\varrho),
$$

which is true for pure states with some $A_{n}$ operators. Here $N_{A}$ is the number of $A_{n}$ operators we consider. If $g(\varrho)$ is convex in density matrices, then

$$
I\left(\left\{A_{n}\right\}_{n=1}^{N_{A}}, \varrho\right) \geqslant g(\varrho)
$$

holds for mixed states, where we define

$$
I\left(\left\{A_{n}\right\}_{n=1}^{N_{A}}, \varrho\right)=\min _{\left\{p_{k},\left|\psi_{k}\right\rangle\right\}} \sum_{k} p_{k} \sum_{n=1}^{N_{A}}\left(\Delta A_{n}\right)_{\psi_{k}}^{2} .
$$

If $g(\varrho)$ is not convex in $\varrho$, then the inequality with a convex roof

$$
I\left(\left\{A_{n}\right\}_{n=1}^{N_{A}}, \varrho\right) \geqslant \min _{\left\{p_{k},\left|\psi_{k}\right\rangle\right\}} \sum_{k} p_{k} g\left(\left|\psi_{k}\right\rangle\right)
$$

still holds.

Proof. The proof is analogous to that of Observation 6.

For a single operator

$$
I(\{A\}, \varrho)=\frac{1}{4} F_{Q}[\varrho, A]
$$

holds. For two or more operators, it is clear that $I\left(\left\{A_{n}\right\}_{n=1}^{N_{A}}, \varrho\right)$ can be larger than the sum of the corresponding QFI terms:

$$
I\left(\left\{A_{n}\right\}_{n=1}^{N_{A}}, \varrho\right) \geqslant \frac{1}{4} \sum_{n=1}^{N_{A}} F_{Q}\left[\varrho, A_{n}\right] .
$$

There are efficient methods to calculate the convex roof in Eq. (114) with semidefinite programming [77]. Calculating the minimum of Eq. (114) for a set of constraints on the expectation values of operators $B_{n}$ is possible with the Hamiltonian

$$
H_{\left\{\lambda_{n}\right\}_{n=1}^{N_{A}},\left\{\mu_{n}\right\}_{n=1}^{N_{c}}}=\sum_{n=1}^{N_{A}}\left(A_{n}^{2}-\lambda_{n} A_{n}\right)-\sum_{n=1}^{N_{c}} \mu_{n} B_{n},
$$

where $N_{c}$ is the number of constraints. In many cases the lower bound on $I\left(\left\{A_{n}\right\}_{n=1}^{N_{A}}, \varrho\right)$ can be obtained, analogously to Eq. (111) as

$$
\min _{\left\{\lambda_{n}\right\}_{n=1}^{N_{A}},\left\{\mu_{n}\right\}_{n=1}^{N_{C}}:\left\{\left\langle B_{n}\right\rangle=b_{n}\right\}_{n=1}^{N_{c}}} \sum_{k=1}^{N_{A}}\left(\Delta A_{k}\right)_{\mid \Psi}^{2}\left\{\lambda_{n}\right\}_{n=1}^{N_{A}}\left\{\left\{\mu_{n}\right\}_{n=1}^{N_{C}}\right\rangle \cdot
$$

In principle, some complications might arise if the ground state of the Hamiltonian given in Eq. (118) is degenerate or due to the fact that the minimization was restricted to pure states [78]. Reference [56] considers a similar problem, but uses the Legendre transform instead of Lagrange multipliers for the case of a single $A_{n}$ operator. The method can straightforwardly be generalized to the case of several $A_{n}$ operators.

Finally, we can obtain a similar relation with a maximization rather than a minimization over the decomposition.

Observation 9. Let us consider a relation

$$
\sum_{n=1}^{N_{A}}\left(\Delta A_{n}\right)_{\varrho}^{2} \leqslant h(\varrho)
$$

which is true for pure states with some $A_{n}$ operators. If $h(\varrho)$ is concave in density matrices, then

$$
R\left(\left\{A_{n}\right\}_{n=1}^{N_{A}}, \varrho\right) \leqslant h(\varrho)
$$

holds for mixed states, where we define via a concave roof the quantity

$$
R\left(\left\{A_{n}\right\}_{n=1}^{N_{A}}, \varrho\right)=\max _{\left\{p_{k},\left|\psi_{k}\right\rangle\right\}} \sum_{k} p_{k} \sum_{n=1}^{N_{A}}\left(\Delta A_{n}\right)_{\psi_{k}}^{2} .
$$

If $h(\varrho)$ is not concave in $\varrho$, the inequality with a concave roof

$$
R\left(\left\{A_{n}\right\}_{n=1}^{N_{A}}, \varrho\right) \leqslant \max _{\left\{p_{k},\left|\psi_{k}\right\rangle\right\}} \sum_{k} p_{k} h\left(\left|\psi_{k}\right\rangle\right)
$$

still holds.

Proof. The proof is analogous to that of Observation 8.

Clearly, for a single operator

$$
R(\{A\}, \varrho)=(\Delta A)_{\varrho}^{2}
$$

holds. It can be shown that if we have only two operators, then $[59,60,77]$

$$
R\left(\left\{A_{1}, A_{2}\right\}, \varrho\right)=\left(\Delta A_{1}\right)_{\varrho}^{2}+\left(\Delta A_{2}\right)_{\varrho}^{2} .
$$

For three observables, $R\left(\left\{A_{1}, A_{2}, A_{2}\right\}\right.$ can be smaller than the sum of the variances

$$
R\left(\left\{A_{1}, A_{2}, A_{2}\right\}, \varrho\right) \leqslant\left(\Delta A_{1}\right)_{\varrho}^{2}+\left(\Delta A_{2}\right)_{\varrho}^{2}+\left(\Delta A_{3}\right)_{\varrho}^{2} .
$$

Let us see a simple application for entanglement detection.

Observation 10. For separable states for $N$ spin- $j$ particles

$$
V\left(\left\{J_{x}, J_{y}, J_{z}\right\}, \varrho\right) \geqslant N j
$$

holds, which has been presented in Ref. [77]. Any state violating the inequality (127) is entangled.

Proof. We know that for pure product states of $N$ spin- $j$ particles we have [75,79-81]

$$
\left(\Delta J_{x}\right)^{2}+\left(\Delta J_{y}\right)^{2}+\left(\Delta J_{z}\right)^{2} \geqslant N j .
$$

Thus, Eq. (127) is true for pure product states. Since $V\left(\left\{J_{x}, J_{y}, J_{z}\right\}, \varrho\right)$ is concave in $\varrho$, it is also true for separable states, which are just mixtures of product states.

The left-hand side of the relation with there variances given in Eq. (128) is not smaller than the left-hand side of the 
criterion with $V\left(\left\{J_{x}, J_{y}, J_{z}\right\}, \varrho\right)$ given in Eq. (127), and in some cases it is larger. Hence, the condition given in Eq. (127) detects all states that are detected as entangled by Eq. (128), and it detects some further states.

\section{METROLOGICAL USEFULNESS AND ENTANGLEMENT CONDITIONS}

In this section we will connect the violation of uncertaintybased entanglement criteria to the metrological usefulness of the quantum state. With these findings, we address an important problem of entanglement theory: even if entanglement is detected, it is not yet sure that the entanglement is useful for some quantum information processing task or quantum metrology [20]. We will discuss first entanglement conditions for two bosonic modes, then entanglement criteria for two spins.

\section{A. Two-mode quantum states}

In this section we will consider continuous variable systems. A bosonic mode can be described by the canonical $x$ and $p$ operators. For coherent states, $|\alpha\rangle$

$$
(\Delta x)^{2}=(\Delta p)^{2}=\frac{1}{2}
$$

holds. For mixtures of coherent states

$$
\varrho_{\mathrm{mc}}=\sum_{k} p_{k}\left|\alpha_{k}\right\rangle\left\langle\alpha_{k}\right|
$$

we have, due to the concavity of the variance and the convexity of the QFI,

$$
(\Delta x)^{2},(\Delta p)^{2} \geqslant \frac{1}{2}, \quad F_{Q}[x, \varrho], F_{Q}[p, \varrho] \leqslant 2 .
$$

Let us now consider a two-mode system with the position and momentum operators $x_{1}, p_{1}, x_{2}, p_{2}$.

Observation 11. For a mixture of products of coherent states $\alpha_{k}^{(l)}$ of the form

$$
\varrho_{\text {sepc }}=\sum_{k} p_{k}\left|\alpha_{k}^{(1)}\right\rangle\left\langle\alpha_{k}^{(1)}|\otimes| \alpha_{k}^{(2)}\right\rangle\left\langle\alpha_{k}^{(2)}\right|
$$

the collective variances of the position and momentum are bounded from below as

$$
\left[\Delta\left(x_{1} \pm x_{2}\right)\right]^{2} \geqslant 1, \quad\left[\Delta\left(p_{1} \pm p_{2}\right)\right]^{2} \geqslant 1 .
$$

Moreover, the QFI for the same operators is bounded from above as

$$
F_{Q}\left[\varrho, p_{1} \pm p_{2}\right] \leqslant 4, \quad F_{Q}\left[\varrho, x_{1} \pm x_{2}\right] \leqslant 4 .
$$

Note that for such states the multivariable Glauber-Sudarshan $P$ function is non-negative [82].

Proof. For a coherent state, for the variances of $x$ and $p$ the relation in Eq. (129) holds. Then for a tensor product of two coherent states we have

$$
\left[\Delta\left(x_{1} \pm x_{2}\right)\right]^{2}=\left[\Delta\left(p_{1} \pm p_{2}\right)\right]^{2}=1 .
$$

Since for pure states the QFI is four times the variance, for a tensor product of two coherent states we have

$$
F_{Q}\left[\varrho, x_{1} \pm x_{2}\right]=F_{Q}\left[\varrho, p_{1} \pm p_{2}\right]=4 .
$$

Then the statement follows from the concavity of the variance and the convexity of the QFI.

Let us now consider entanglement detection in such systems with uncertainty relations. A well-known entanglement criterion is $[83,84]$

$$
\left[\Delta\left(x_{1}+x_{2}\right)\right]^{2}+\left[\Delta\left(p_{1}-p_{2}\right)\right]^{2} \geqslant 2 .
$$

If a quantum state violates Eq. (137), then it is entangled.

Next, let us connect the violation of Eq. (137) to the metrological properties of the quantum state.

Observation 12. For a two-mode state, the following uncertainty relation holds:

$$
\begin{aligned}
& {\left[\Delta\left(x_{1}+x_{2}\right)\right]^{2}+\left[\Delta\left(p_{1}-p_{2}\right)\right]^{2}} \\
& \quad \geqslant 4 / F_{Q}\left[\varrho, p_{1}+p_{2}\right]+4 / F_{Q}\left[\varrho, x_{1}-x_{2}\right] .
\end{aligned}
$$

As a consequence of Eq. (138), states violating the entanglement condition given in Eq. (137) are metrologically more useful than states of the form given in Eq. (132), i.e., bipartite states with a non-negative multivariable Glauber-Sudarshan $P$ function.

Proof. We start from the relations

$$
\begin{aligned}
& {\left[\Delta\left(x_{1}+x_{2}\right)\right]^{2} F_{Q}\left[\varrho, p_{1}+p_{2}\right] \geqslant 4,} \\
& {\left[\Delta\left(p_{1}-p_{2}\right)\right]^{2} F_{Q}\left[\varrho, x_{1}-x_{2}\right] \geqslant 4,}
\end{aligned}
$$

which are the applications of Eq. (38). Then in both inequalities of Eq. (139) we divide by the term containing the QFI. Finally, we sum the two resulting inequalities.

Next, we will show that violating the condition given in Eq. (137) implies metrological usefulness compared to a special class of separable states. Due to Eq. (138), the violation of the entanglement criterion given in Eq. (137) implies the violation of one of the inequalities of Eq. (134). Thus, violation of the uncertainty relation-based entanglement condition also means that the state has larger metrological usefulness than states of the type given in Eq. (132).

Note, however, that we did not prove that violating the entanglement condition given in Eq. (137) leads to larger metrological usefulness than that of separable states in general, since even for pure product states $F_{Q}\left[\varrho, x_{1} \pm x_{2}\right]$ or $F_{Q}\left[\varrho, p_{1} \pm p_{2}\right]$ can be arbitrarily large for two bosonic modes.

\section{B. Spin systems}

Next, we will consider a system of two spins. For this case we can show that if entanglement is detected by a wellknown entanglement condition, then the state is more useful for metrology than a certain subset of separable states.

Let us see first a well-known entanglement conditions for two spins [79]. For separable states

$$
\begin{gathered}
{\left[\Delta\left(J_{x}^{(1)}+J_{x}^{(2)}\right)\right]^{2}+\left[\Delta\left(J_{y}^{(1)}+J_{y}^{(2)}\right)\right]^{2}} \\
+\left[\Delta\left(J_{z}^{(1)}+J_{z}^{(2)}\right)\right]^{2} \geqslant j_{1}+j_{2}
\end{gathered}
$$

holds. Any state violating Eq. (140) is entangled. Note that this is the same condition as Eq. (128) for the special case of two qudits.

Next, we need a similar relation for the QFI. For that, let us consider a special class of mixed states, a mixture of spin 
coherent states of a spin- $j$ particle given as

$$
\varrho_{\mathrm{msc}}=\sum_{k} p_{k}\left|s_{k}\right\rangle\left\langle s_{k}\right| \text {. }
$$

Here the spin-coherent states are defined similarly as in Eq. (83). It is easy to see that for such states

$$
\sum_{l=x, y, z} F_{Q}\left[\varrho, j_{l}\right] \leqslant 4 j
$$

holds, where the inequality is saturated for all pure spin coherent states. The maximum of the left-hand side of Eq. (142) for general quantum states is $4 j(j+1)$. We add that for spincoherent states

$$
F_{Q}\left[\varrho, j_{l}\right] \leqslant 2 j
$$

also holds for $l=x, y, z$, where the inequality is saturated for $|+j\rangle_{k}$ for $k \neq l$. The maximum of the left-hand side of Eq. (143) for general quantum states is $4 j^{2}$.

Let us now move to bipartite systems.

Observation 13. For a mixture of products of spincoherent states $\left|s_{k}^{(l)}\right\rangle$ of the form

$$
\varrho_{\text {sepsc }}=\sum_{k} p_{k}\left|s_{k}^{(1)}\right\rangle\left\langle s_{k}^{(1)}|\otimes| s_{k}^{(2)}\right\rangle\left\langle s_{k}^{(2)}\right|,
$$

the relation with the sum of three QFI terms

$$
\begin{gathered}
F_{Q}\left[\varrho, J_{x}^{(1)} \pm J_{x}^{(2)}\right]+F_{Q}\left[\varrho, J_{y}^{(1)} \pm J_{y}^{(2)}\right] \\
+F_{Q}\left[\varrho, J_{z}^{(1)} \pm J_{z}^{(2)}\right] \leqslant 4\left(j_{1}+j_{2}\right)
\end{gathered}
$$

holds.

Proof. This is just a generalization of the statements presented in Refs. [21,22]. For a pure product of spin-coherent states, for the left-hand side of Eq. (145) we have

$$
4\left[\sum_{l=x, y, z}\left(\Delta J_{l}^{(1)}\right)^{2}+\sum_{l=x, y, z}\left(\Delta J_{l}^{(2)}\right)^{2}\right]=4\left(j_{1}+j_{2}\right) \text {. }
$$

Due to the convexity of the QFI, the left-hand side of Eq. (145) cannot be larger than the right-hand side even for mixed states.

A state violating the inequality given in Eq. (145) is more useful metrologically than a mixture of products of spincoherent states if we consider not a single metrological task, but the three tasks corresponding to the three QFI terms in Eq. (145).

Next, we will show how the violation of Eq. (140) implies metrological usefulness.

Observation 14. For a bipartite quantum state

$$
\begin{aligned}
8 & \sum_{l=x, y, z}\left[\Delta\left(J_{l}^{(1)}+J_{l}^{(2)}\right)\right]^{2} \\
& +\sum_{l=x, y, z} F_{Q}\left[\varrho, J_{l}^{(1)}-J_{l}^{(2)}\right] \geqslant 12\left(j_{1}+j_{2}\right)
\end{aligned}
$$

holds. Here $J_{l}^{(n)}$ for $l=x, y, z$ are spin operators acting on the two subsystems, and $j_{n}$ are the spins of the two parties.

As a consequence of Eq. (147), states violating the entanglement condition in Eq. (140) are metrologically more useful than mixtures of products of spin-coherent states given in Eq. (144), which are a subset of separable states, for the combination of the three metrological tasks corresponding to the three QFI terms in Eq. (147). For the case $j_{1}=j_{2}=1 / 2$, this also means that they are more useful than separable states.

Proof. We start from the uncertainty relations for the two parties

$$
\left(\Delta J_{x}^{(n)}\right)^{2}+\left(\Delta J_{y}^{(n)}\right)^{2}+\left(\Delta J_{z}^{(n)}\right)^{2} \geqslant j_{n},
$$

where $n=1,2$. For pure states of spin-1/2 particles, the equality holds. Then we need the fact that

$$
\begin{gathered}
F_{Q}\left[\varrho, J_{x}^{(1)}-J_{x}^{(2)}\right] / 4+\left[\Delta\left(J_{y}^{(1)}+J_{y}^{(2)}\right)\right]^{2} \\
+\left[\Delta\left(J_{z}^{(1)}+J_{z}^{(2)}\right)\right]^{2} \geqslant j_{1}+j_{2}
\end{gathered}
$$

is valid for any quantum state. This can be seen knowing that it is true for pure states, i.e.,

$$
\begin{gathered}
{\left[\Delta\left(J_{x}^{(1)}-J_{x}^{(2)}\right)\right]^{2}+\left[\Delta\left(J_{y}^{(1)}+J_{y}^{(2)}\right)\right]^{2}} \\
+\left[\Delta\left(J_{z}^{(1)}+J_{z}^{(2)}\right)\right]^{2} \geqslant j_{1}+j_{2},
\end{gathered}
$$

which can be proved similarly to Eq. (140). Then the mixed state condition follows from ideas of Sec. VIB. Using Eq. (149), and all the inequalities obtained from it after permuting $x, y$, and $z$, and adding these inequalities, we arrive at Eq. (147).

Let us see the second part of the observation. If Eq. (140) is violated, then based on Eq. (147)

$$
\sum_{l=x, y, z} F_{Q}\left[\varrho, J_{l}^{(1)}-J_{l}^{(2)}\right]>4\left(j_{1}+j_{2}\right)
$$

must hold. We know that for a mixture of products of spincoherent states the inequality given in Eq. (145) holds. Thus, the quantum states violating the entanglement condition given in Eq. (140) are more useful for metrology than states of the form Eq. (144).

Let us examine Eq. (147) for SU(2) singlet states. For such states,

$$
\left\langle\left(J_{l}^{(1)}+J_{l}^{(2)}\right)^{2}\right\rangle=0
$$

for $l=x, y, z$. Hence, for such states the first sum in Eq. (147) is zero, and

$$
\sum_{l=x, y, z} F_{Q}\left[\varrho, J_{l}^{(1)}-J_{l}^{(2)}\right] \geqslant 12\left(j_{1}+j_{2}\right) .
$$

Hence singlet states violate Eq. (145) with the choice of "-" for all the three terms.

Singlets are invariant under Hamiltonians of the type

$$
H_{0}=B_{0}\left(J_{l}^{(1)}+J_{l}^{(2)}\right),
$$

which describes the effect of homogeneous magnetic fields, where $B_{0}$ is a constant proportional to the strength of the homogeneous magnetic field. However, singlet states are sensitive to field gradients [85-87].

\section{CONCLUSIONS}

We studied various relations obtained from the Schrödinger-Robertson uncertainty after an optimization over all the possible decompositions of the density matrix 
is applied. Using convex roofs over decompositions, we rederived the inequality presented in Ref. [41] and gained insights concerning the Cramér-Rao bound. We also used concave roofs to obtain improvements on the RobertsonSchrödinger uncertainty relation. Finally, using similar techniques, we introduced inequalities with variances and the QFI. Similar techniques might make it possible to obtain inequalities for variances and the QFI from further inequalities for variances [88].

Independently from our work, the convex-roof property of the QFI has been used to derive uncertainty relations by Chiew and Gessner [89].

\section{ACKNOWLEDGMENTS}

We thank I. Apellaniz, R. Demkowicz-Dobrzański, P. Hyllus, O. Gühne, M. Kleinmann, J. Kołodyński, J. Siewert, A. Smerzi, Sz. Szalay, R. Trényi, and G. Vitagliano for stimulating discussions. We acknowledge the support of the EU (COST Action CA15220, QuantERA CEBBEC, QuantERA MENTA), the Spanish MCIU (Grant No. PCI2018092896), the Spanish Ministry of Science, Innovation and Universities and the European Regional Development Fund FEDER through Grant No. PGC2018-101355-B-I00 (MCIU/AEI/FEDER, EU), the Basque Government (Grant No. IT986-16), and the National Research, Development and Innovation Office NKFIH (Grants No. K124351, No. KH129601). We thank the "Frontline" Research Excellence Programme of the NKFIH (Grant No. KKP133827). G.T. is thankful for a Bessel Research Award from the Humboldt Foundation.

\section{APPENDIX A: DERIVATION OF EQ. (29)}

We derive Eq. (29) from knowing that the RobertsonSchrödinger inequality given in Eq. (23) holds for all $\varrho_{k}$ components.

Let us consider the inequality

$$
\left(\sum_{k} p_{k} a_{k}\right)\left(\sum_{k} p_{k} b_{k}\right) \geqslant\left(\sum_{k} p_{k} \sqrt{a_{k} b_{k}}\right)^{2},
$$

where $a_{k}, b_{k} \geqslant 0$. It can be proved as follows. It can be rewritten as

$$
\sum_{k, l} p_{k} p_{l}\left(a_{k} b_{l}+a_{l} b_{k}\right) \geqslant \sum_{k, l} p_{k} p_{l} 2 \sqrt{a_{k} a_{l} b_{l} b_{k}} .
$$

Term by term, the left-hand side is larger or equal to the righthand side, since $\left(\sqrt{a_{k} b_{l}}-\sqrt{a_{l} b_{k}}\right)^{2} \geqslant 0$. If additionally

$$
a_{k} b_{k} \geqslant c_{k}^{2}
$$

holds for all $k$, then we arrive at

$$
\left(\sum_{k} p_{k} a_{k}\right)\left(\sum_{k} p_{k} b_{k}\right) \geqslant\left(\sum_{k} p_{k}\left|c_{k}\right|\right)^{2} .
$$

Note that Eq. (A1), and hence Eq. (A4) can be saturated only if

$$
a_{k}=a_{l}, \quad b_{k}=b_{l},
$$

hold for all $k, l$. Finally, in order to have equality in Eq. (A4), we also need that Eq. (A3) is saturated for all $k$. In this case, all $c_{k}$ must be equal to each other.

The inequality in Eq. (29) can be derived from the relation in Eq. (A4) knowing that the uncertainty relation given in Eq. (23) holds for the $\varrho_{k}$ components in a decomposition given in Eq. (21). We need to introduce $a_{k}=(\Delta A)_{\varrho_{k}}^{2}, b_{k}=(\Delta B)_{\varrho_{k}}^{2}$, and $c_{k}=\frac{1}{2} L_{\varrho_{k}}$. If we use an inequality analogous to Eq. (29) for pure-state decompositions given in Eq. (15), then we need $a_{k}=(\Delta A)_{\psi_{k}}^{2}, b_{k}=(\Delta B)_{\psi_{k}}^{2}$, and $c_{k}=\frac{1}{2} L_{\psi_{k}}$.

\section{APPENDIX B: NUMERICAL CALCULATION OF CONCAVE ROOFS}

In this Appendix we will discuss how to compute concave roofs numerically. Concave roofs can be computed by brute force optimization. We will now describe a simple numerical method to find such bounds. Other method is similar to the one in Ref. [90], as it is also based on the purification of the mixed state. The statements also hold for concave roofs, after trivial changes.

In order to obtain concave roofs, we have to carry out a numerical optimization over all decompositions of the density matrix. First, let us consider decompositions to pure states given in Eq. (15). Let us define the purification of $\varrho$ [91],

$$
\left|\Psi_{p}\right\rangle=\sum_{k} \sqrt{p_{k}}\left|\psi_{k}\right\rangle_{S} \otimes|k\rangle_{A},
$$

where $S$ denotes the system, $A$ is the ancilla, and for this state,

$$
\operatorname{Tr}_{A}\left(\left|\Psi_{p}\right\rangle\left\langle\Psi_{p}\right|\right)=\varrho
$$

holds. One of the purifications that is easy to write is the one based on the eigendecomposition of the density matrix, and for that we need an ancilla that has the same size as the system. For other purifications, we might need an ancilla larger than the system. The dimension of the ancilla equals the number of pure subensembles we consider.

Since all purifications can be obtained from each other by a unitary acting on the ancilla, we arrive at the following. For any quantity $Q(\sigma)$, which is a function of a mixed state $\sigma$, we can write the concave roof as an optimum over the decompositions as

$$
\begin{aligned}
& \max _{\left\{p_{k},\left|\psi_{k}\right\rangle\right\}} \sum_{k} p_{k} Q\left(\left|\psi_{k}\right\rangle\left\langle\psi_{k}\right|\right) \\
& =\max _{U_{A}} \sum_{k}\left\langle v_{k} \mid v_{k}\right\rangle Q\left(\left|v_{k}\right\rangle\left\langle v_{k}\right| /\left\langle v_{k} \mid v_{k}\right\rangle\right),
\end{aligned}
$$

where the maximization is over unitaries acting on the ancilla and we defined the unnormalized vectors as

$$
\left|v_{k}\right\rangle=\left\langle\left. k\right|_{A} U_{A} \mid \Psi_{p}\right\rangle .
$$

Note that one can show that

$$
\varrho=\sum_{k}\left|v_{k}\right\rangle\left\langle v_{k}\right|
$$

These ideas can be extended to mixed-state decompositions given in Eq. (21) as follows. Similarly to Sec. V, we consider not only pure-state decompositions, but also 
mixed-state decompositions in which the mixed components are mixtures of some of the $\left|v_{k}\right\rangle$. We can extend this method to optimize over all mixed-state decompositions as follows:

$$
\begin{aligned}
& \max _{\left\{K_{l}\right\}} \max _{\left\{p_{l}, \rho_{l}\right\}} \sum_{l} p_{l} Q\left(\varrho_{l}\right) \\
& \quad=\max _{\left\{K_{l}\right\}} \max _{U_{A}} \sum_{l} \operatorname{Tr}\left(\sigma_{l}\right) Q\left[\sigma_{l} / \operatorname{Tr}\left(\sigma_{l}\right)\right],
\end{aligned}
$$

where unnormalized states are

$$
\sigma_{l}=\sum_{k \in K_{l}}\left\langle\left. k\right|_{A} U_{A} \mid \Psi_{p}\right\rangle\left\langle\Psi_{p}\left|U_{A}^{\dagger}\right| k\right\rangle_{A} .
$$

The probabilities and the normalized states of the decomposition are given as

$$
p_{k}=\operatorname{Tr}\left(\sigma_{k}\right), \quad \varrho_{k}=\sigma_{k} / p_{k} .
$$

Here the basis states are distributed into sets $K_{l}$. For instance, $K_{1}=1, K_{2}=2$, and $K_{3}=3$ correspond to looking for a purestate decomposition. $K_{1}=\{1,2\}$ and $K_{2}=3$ correspond to looking for a mixture of a rank-2 mixed state and a pure state. In Fig. 1(b) the results are shown for using the method above where both the system and the ancilla have a dimension $d=3$.

Looking for the unitary that leads to the maximum can be done with a multivariable search. We developed a simple algorithm based on a random search and improving the best random guess by small local changes. The local changes are also random, and they are accepted if they increase the quantity to be maximized. A computer program based on such an algorithm is incorporated in the newest version of the QUBIT4MATLAB package [92]. Such random optimization has already been used to look for the maximum of an operator expectation value for separable states in the same program package.

Reference [77] presents a method that provides good upper bounds of concave roofs based on semidefinite programming, but it works only for small systems of a couple of qubits. The result of this procedure is larger or equal to the true bound and thus can be used to evaluate whether the bound found with the brute force search is optimal.

Calculating the convex roof is similar, only the maximization has to be replaced by minimization in Eq. (B3).
[1] V. Giovannetti, S. Lloyd, and L. Maccone, Quantum-enhanced measurements: Beating the standard quantum limit, Science 306, 1330 (2004).

[2] M. G. A. Paris, Quantum estimation for quantum technology, Int. J. Quantum Inform. 07, 125 (2009).

[3] R. Demkowicz-Dobrzanski, M. Jarzyna, and J. Kolodynski, Chapter four-Quantum limits in optical interferometry, Prog. Opt. 60, 345 (2015).

[4] L. Pezze and A. Smerzi, Quantum theory of phase estimation, in Atom Interferometry (Proc. Int. School of Physics 'Enrico Fermi', Course 188, Varenna), edited by G. Tino and M. Kasevich (IOS Press, Amsterdam, 2014), pp. 691-741.

[5] C. Helstrom, Quantum Detection and Estimation Theory (Academic Press, New York, 1976).

[6] A. Holevo, Probabilistic and Statistical Aspects of Quantum Theory (North-Holland, Amsterdam, 1982).

[7] S. L. Braunstein and C. M. Caves, Statistical Distance and the Geometry of Quantum States, Phys. Rev. Lett. 72, 3439 (1994).

[8] S. L. Braunstein, C. M. Caves, and G. J. Milburn, Generalized uncertainty relations: Theory, examples, and Lorentz invariance, Ann. Phys. 247, 135 (1996).

[9] S. F. Huelga, C. Macchiavello, T. Pellizzari, A. K. Ekert, M. B. Plenio, and J. I. Cirac, Improvement of Frequency Standards with Quantum Entanglement, Phys. Rev. Lett. 79, 3865 (1997).

[10] B. Escher, R. de Matos Filho, and L. Davidovich, General framework for estimating the ultimate precision limit in noisy quantum-enhanced metrology, Nat. Phys. 7, 406 (2011).

[11] R. Demkowicz-Dobrzański, J. Kołodyński, and M. Guţă, The elusive Heisenberg limit in quantum-enhanced metrology, Nat. Commun. 3, 1063 (2012).

[12] D. Leibfried, M. Barrett, T. Schaetz, J. Britton, J. Chiaverini, W. Itano, J. Jost, C. Langer, and D. Wineland, Toward Heisenberg-limited spectroscopy with multiparticle entangled states, Science 304, 1476 (2004).
[13] M. Napolitano, M. Koschorreck, B. Dubost, N. Behbood, R. Sewell, and M. W. Mitchell, Interaction-based quantum metrology showing scaling beyond the Heisenberg limit, Nature (London) 471, 486 (2011).

[14] M. F. Riedel, P. Böhi, Y. Li, T. W. Hänsch, A. Sinatra, and P. Treutlein, Atom-chip-based generation of entanglement for quantum metrology, Nature (London) 464, 1170 (2010).

[15] C. Gross, T. Zibold, E. Nicklas, J. Esteve, and M. K. Oberthaler, Nonlinear atom interferometer surpasses classical precision limit, Nature (London) 464, 1165 (2010).

[16] H. Grote, K. Danzmann, K. L. Dooley, R. Schnabel, J. Slutsky, and H. Vahlbruch, First Long-Term Application of Squeezed States of Light in a Gravitational-Wave Observatory, Phys. Rev. Lett. 110, 181101 (2013).

[17] J. Aasi, J. Abadie, B. P. Abbott, R. Abbott, and T. D. Abbott, Enhanced sensitivity of the LIGO gravitational wave detector by using squeezed states of light, Nat. Photon. 7, 613 (2013).

[18] R. Demkowicz-Dobrzański, K. Banaszek, and R. Schnabel, Fundamental quantum interferometry bound for the squeezed-light-enhanced gravitational wave detector GEO 600, Phys. Rev. A 88, 041802(R) (2013).

[19] G. Tóth and I. Apellaniz, Quantum metrology from a quantum information science perspective, J. Phys. A: Math. Theor. 47, 424006 (2014).

[20] L. Pezzé and A. Smerzi, Entanglement, Nonlinear Dynamics, and the Heisenberg Limit, Phys. Rev. Lett. 102, 100401 (2009).

[21] P. Hyllus, W. Laskowski, R. Krischek, C. Schwemmer, W. Wieczorek, H. Weinfurter, L. Pezzé, and A. Smerzi, Fisher information and multiparticle entanglement, Phys. Rev. A 85, 022321 (2012)

[22] G. Tóth, Multipartite entanglement and high-precision metrology, Phys. Rev. A 85, 022322 (2012). 
[23] S. Szalay, k-stretchability of entanglement, and the duality of k-separability and k-producibility, Quantum 3, 204 (2019).

[24] G. Tóth, Stretching the limits of multiparticle entanglement, Quantum Views 4, 30 (2020).

[25] Z. Ren, W. Li, A. Smerzi, and M. Gessner, Metrological Detection of Multipartite Entanglement from Young Diagrams, Phys. Rev. Lett. 126, 080502 (2021).

[26] B. Lücke, M. Scherer, J. Kruse, L. Pezzé, F. Deuretzbacher, P. Hyllus, J. Peise, W. Ertmer, J. Arlt, L. Santos, A. Smerzi, and C. Klempt, Twin matter waves for interferometry beyond the classical limit, Science 334, 773 (2011).

[27] R. Krischek, C. Schwemmer, W. Wieczorek, H. Weinfurter, P. Hyllus, L. Pezzé, and A. Smerzi, Useful Multiparticle Entanglement and Sub-Shot-Noise Sensitivity in Experimental Phase Estimation, Phys. Rev. Lett. 107, 080504 (2011).

[28] H. Strobel, W. Muessel, D. Linnemann, T. Zibold, D. B. Hume, L. Pezzé, A. Smerzi, and M. K. Oberthaler, Fisher information and entanglement of non-Gaussian spin states, Science 345, 424 (2014).

[29] F. Fröwis and W. Dür, Stable Macroscopic Quantum Superpositions, Phys. Rev. Lett. 106, 110402 (2011).

[30] F. Fröwis and W. Dür, Are Cloned Quantum States Macroscopic?, Phys. Rev. Lett. 109, 170401 (2012).

[31] A. del Campo, I. L. Egusquiza, M. B. Plenio, and S. F. Huelga, Quantum Speed Limits in Open System Dynamics, Phys. Rev. Lett. 110, 050403 (2013).

[32] M. M. Taddei, B. M. Escher, L. Davidovich, and R. L. de Matos Filho, Quantum Speed Limit for Physical Processes, Phys. Rev. Lett. 110, 050402 (2013).

[33] A. Smerzi, Zeno Dynamics, Indistinguishability of State, and Entanglement, Phys. Rev. Lett. 109, 150410 (2012).

[34] F. Schäfer, I. Herrera, S. Cherukattil, C. Lovecchio, F. Cataliotti, F. Caruso, and A. Smerzi, Experimental realization of quantum zeno dynamics, Nat. Commun. 5, 3194 (2014).

[35] C. Schwemmer, G. Tóth, A. Niggebaum, T. Moroder, D. Gross, O. Gühne, and H. Weinfurter, Experimental Comparison of Efficient Tomography Schemes for a Six-Qubit State, Phys. Rev. Lett. 113, 040503 (2014).

[36] C. Schwemmer, L. Knips, D. Richart, H. Weinfurter, T. Moroder, M. Kleinmann, and O. Gühne, Systematic Errors in Current Quantum State Tomography Tools, Phys. Rev. Lett. 114, 080403 (2015).

[37] G. Tóth and D. Petz, Extremal properties of the variance and the quantum Fisher information, Phys. Rev. A 87, 032324 (2013).

[38] S. Yu, Quantum fisher information as the convex roof of variance, arXiv:1302.5311.

[39] R. Horodecki, P. Horodecki, M. Horodecki, and K. Horodecki, Quantum entanglement, Rev. Mod. Phys. 81, 865 (2009).

[40] O. Gühne and G. Tóth, Entanglement detection, Phys. Rep. 474, 1 (2009).

[41] F. Fröwis, R. Schmied, and N. Gisin, Tighter quantum uncertainty relations following from a general probabilistic bound, Phys. Rev. A 92, 012102 (2015).

[42] A. Kholevo, A generalization of the Rao-Cramer inequality, Theory Probab. Appl. 18, 359 (1974).

[43] W. Zhong, X. M. Lu, X. X. Jing, and X. Wang, Optimal condition for measurement observable via error-propagation, J. Phys. A: Math. Theor. 47, 385304 (2014).

[44] W. Heisenberg, Über den anschaulichen Inhalt der quantentheoretischen Kinematik und Mechanik, Z. Phys. 43, 172 (1927).
[45] H. P. Robertson, The uncertainty principle, Phys. Rev. 34, 163 (1929).

[46] L. Mandelstam and I. Tamm, The uncertainty relation between energy and time in non-relativistic quantum mechanics, J. Phys. (USSR) 9, 249 (1945).

[47] G. N. Fleming, A unitarity bound on the evolution of nonstationary states, Nuovo Cimento A 16, 232 (1973).

[48] A. Uhlmann, An energy dispersion estimate, Phys. Lett. A 161, 329 (1992).

[49] F. Fröwis, Kind of entanglement that speeds up quantum evolution, Phys. Rev. A 85, 052127 (2012).

[50] A. Sørensen, L.-M. Duan, J. Cirac, and P. Zoller, Many-particle entanglement with Bose-Einstein condensates, Nature (London) 409, 63 (2001).

[51] M. Gessner, A. Smerzi, and L. Pezzè, Metrological Nonlinear Squeezing Parameter, Phys. Rev. Lett. 122, 090503 (2019).

[52] E. Schrödinger, Zum Heisenbergschen Unschärfeprinzip, Sitzungsber. Preuss. Akad. Wiss., Phys. Math. Kl. 14, 296 (1930).

[53] D. Petz, Quantum Information Theory and Quantum Statistics (Springer, Berlin, 2008).

[54] A. T. Rezakhani, M. Hassani, and S. Alipour, Continuity of the quantum Fisher information, Phys. Rev. A 100, 032317 (2019).

[55] R. Augusiak, J. Kołodyński, A. Streltsov, M. N. Bera, A. Acín, and M. Lewenstein, Asymptotic role of entanglement in quantum metrology, Phys. Rev. A 94, 012339 (2016).

[56] I. Apellaniz, M. Kleinmann, O. Gühne, and G. Tóth, Optimal witnessing of the quantum Fisher information with few measurements, Phys. Rev. A 95, 032330 (2017).

[57] Y. Akbari-Kourbolagh and M. Azhdargalam, Entanglement criterion for multipartite systems based on quantum Fisher information, Phys. Rev. A 99, 012304 (2019).

[58] G. Tóth, Lower bounds on the quantum Fisher information based on the variance and various types of entropies, arXiv:1701.07461.

[59] Z. Léka and D. Petz, Some decompositions of matrix variances, Probab. Math. Statist. 33, 191 (2013).

[60] D. Petz and D. Virosztek, A characterization theorem for matrix variances, Acta Sci. Math. (Szeged) 80, 681 (2014).

[61] A. Fujiwara and H. Imai, A fibre bundle over manifolds of quantum channels and its application to quantum statistics, J. Phys. A: Math. Theor. 41, 255304 (2008).

[62] I. Marvian, Coherence distillation machines are impossible in quantum thermodynamics, Nat. Commun. 11, 25 (2020).

[63] Decomposition to mixed states also appears in entanglement theory; e.g., see M. Hofmann, T. Moroder, and O. Gühne, Analytical characterization of the genuine multiparticle negativity, J. Phys. A: Math. Theor. 47, 155301 (2014).

[64] S. Luo, Heisenberg uncertainty relation for mixed states, Phys. Rev. A 72, 042110 (2005).

[65] S. Furuichi, Schrödinger uncertainty relation with WignerYanase skew information, Phys. Rev. A 82, 034101 (2010).

[66] S. Yu and C. H. Oh, Robertson-Schroedinger uncertainty relation refined by skew information, arXiv:1303.6404.

[67] L. Maccone and A. K. Pati, Stronger Uncertainty Relations for all Incompatible Observables, Phys. Rev. Lett. 113, 260401 (2014).

[68] L. Pezzè, A. Smerzi, M. K. Oberthaler, R. Schmied, and P. Treutlein, Quantum metrology with nonclassical states of atomic ensembles, Rev. Mod. Phys. 90, 035005 (2018). 
[69] M. Hotta and M. Ozawa, Quantum estimation by local observables, Phys. Rev. A 70, 022327 (2004).

[70] B. M. Escher, Quantum noise-to-sensibility ratio, arXiv:1212.2533.

[71] G. Tóth, T. Vértesi, P. Horodecki, and R. Horodecki, Activating Hidden Metrological Usefulness, Phys. Rev. Lett. 125, 020402 (2020).

[72] K. Zyczkowski and H.-J. Sommers, Induced measures in the space of mixed quantum states, J. Phys. A: Math. Gen. 34, 7111 (2001).

[73] Q. Y. He, S.-G. Peng, P. D. Drummond, and M. D. Reid, Planar quantum squeezing and atom interferometry, Phys. Rev. A 84, 022107 (2011).

[74] A. S. Sørensen and K. Mølmer, Entanglement and Extreme Spin Squeezing, Phys. Rev. Lett. 86, 4431 (2001).

[75] G. Vitagliano, P. Hyllus, I. L. Egusquiza, and G. Tóth, Spin Squeezing Inequalities for Arbitrary Spin, Phys. Rev. Lett. 107, 240502 (2011).

[76] V. Giovannetti, S. Mancini, D. Vitali, and P. Tombesi, Characterizing the entanglement of bipartite quantum systems, Phys. Rev. A 67, 022320 (2003).

[77] G. Tóth, T. Moroder, and O. Gühne, Evaluating Convex Roof Entanglement Measures, Phys. Rev. Lett. 114, 160501 (2015).

[78] If the ground state of Eq. (118) is degenerate then we can break the degeneracy with additional operators. Note also that Eq. (119) works based on optimizing over pure states only. However, for some $\left\{\left\langle B_{n}\right\rangle=b_{n}\right\}$ constraints there might not be a corresponding pure state. Thus, when we plot $\sum_{n}\left\langle A_{n}^{2}\right\rangle$ as a function of $\left\langle A_{n}\right\rangle$ and $\left\langle B_{n}\right\rangle$ from results of the optimization over pure states, we have to construct the convex hull from below. Then we can obtain the function $f$ giving the minimum as $\left(\sum_{n}\left\langle A_{n}^{2}\right\rangle\right)_{\min }=f\left(\left\{\left\langle A_{n}\right\rangle\right\}_{n=1}^{N_{A}},\left\{\left\langle B_{n}\right\rangle\right\}_{n=1}^{N_{c}}\right)$ that is valid even for mixed states. We can then compute the minimal variance based on this function rather than using Eq. (119).

[79] G. Tóth, Entanglement detection in optical lattices of bosonic atoms with collective measurements, Phys. Rev. A 69, 052327 (2004).

[80] G. Tóth, C. Knapp, O. Gühne, and H. J. Briegel, Optimal Spin Squeezing Inequalities Detect Bound Entanglement in Spin Models, Phys. Rev. Lett. 99, 250405 (2007).

[81] G. Tóth and M. W. Mitchell, Generation of macroscopic singlet states in atomic ensembles, New J. Phys. 12, 053007 (2010).

[82] E. Agudelo, J. Sperling, and W. Vogel, Quasiprobabilities for multipartite quantum correlations of light, Phys. Rev. A 87, 033811 (2013).
[83] L.-M. Duan, G. Giedke, J. I. Cirac, and P. Zoller, Inseparability Criterion for Continuous Variable Systems, Phys. Rev. Lett. 84, 2722 (2000).

[84] R. Simon, Peres-Horodecki Separability Criterion for Continuous Variable Systems, Phys. Rev. Lett. 84, 2726 (2000).

[85] H. Cable and G. A. Durkin, Parameter Estimation with Entangled Photons Produced by Parametric Down-Conversion, Phys. Rev. Lett. 105, 013603 (2010).

[86] I. Urizar-Lanz, P. Hyllus, I. L. Egusquiza, M. W. Mitchell, and G. Tóth, Macroscopic singlet states for gradient magnetometry, Phys. Rev. A 88, 013626 (2013).

[87] N. Behbood, F. Martin Ciurana, G. Colangelo, M. Napolitano, G. Tóth, R. J. Sewell, and M. W. Mitchell, Generation of Macroscopic Singlet States in a Cold Atomic Ensemble, Phys. Rev. Lett. 113, 093601 (2014).

[88] L. Dammeier, R. Schwonnek, and R. F. Werner, Uncertainty relations for angular momentum, New J. Phys. 17, 093046 (2015).

[89] S.-H. Chiew and M. Gessner, Improving sum uncertainty relations with the quantum Fisher information, Phys. Rev. Research 4, 013076 (2022).

[90] B. Röthlisberger, J. Lehmann, and D. Loss, Numerical evaluation of convex-roof entanglement measures with applications to spin rings, Phys. Rev. A 80, 042301 (2009).

[91] L. P. Hughston, R. Jozsa, and W. K. Wootters, A complete classification of quantum ensembles having a given density matrix, Phys. Lett. A 183, 14 (1993).

[92] The names of the functions relevant for this publication are concroof.m and example_concroof.m, respectively. The program package is available at MATLAB CENTRAL at http:// www.mathworks.com/matlabcentral/. The 3.0 version of the package is described in G. Tóth, QUBIT4MATLAB V3.0: A program package for quantum information science and quantum optics for MATLAB, Comput. Phys. Commun. 179, 430 (2008).

Correction: Equations (47) and (48) contained errors and have been fixed. Minor wording changes have been made in the sentence above Eq. (47) and in the eighth sentence of the first full paragraph below Eq. (73). Equation (B6) was incomplete and has been set right.

Second Correction: The previously implemented correction to Eq. (B6) did not reflect the intended change and has been rectified. 